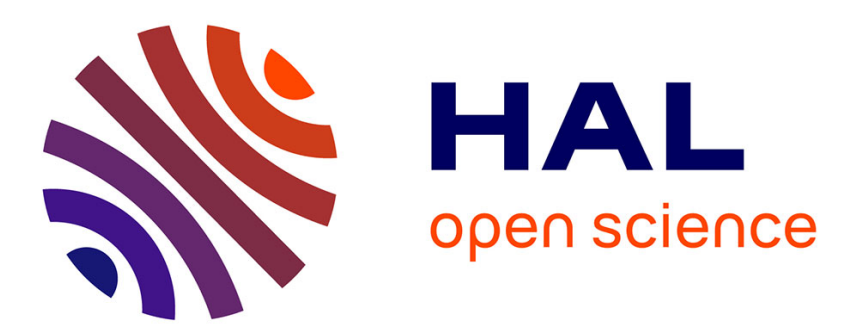

\title{
A posteriori tests of subgrid-scale models in strongly anisothermal turbulent flows
}

Dorian Dupuy, Adrien Toutant, F. Bataille

\section{To cite this version:}

Dorian Dupuy, Adrien Toutant, F. Bataille. A posteriori tests of subgrid-scale models in strongly anisothermal turbulent flows. Physics of Fluids, 2019, 31 (6), pp.065113. 10.1063/1.5098389 . hal02801845

\section{HAL Id: hal-02801845 \\ https://hal.science/hal-02801845}

Submitted on 5 Jun 2020

HAL is a multi-disciplinary open access archive for the deposit and dissemination of scientific research documents, whether they are published or not. The documents may come from teaching and research institutions in France or abroad, or from public or private research centers.
L'archive ouverte pluridisciplinaire HAL, est destinée au dépôt et à la diffusion de documents scientifiques de niveau recherche, publiés ou non, émanant des établissements d'enseignement et de recherche français ou étrangers, des laboratoires publics ou privés. 


\title{
A posteriori tests of subgrid-scale models in strongly anisothermal turbulent flows
}

\author{
Dorian Dupuy $^{1}$, Adrien Toutant*1, and Françoise Bataille ${ }^{1}$ \\ ${ }^{1}$ PROMES CNRS, Université de Perpignan Via Domitia, Rambla de la thermodynamique, \\ Tecnosud, 66100 Perpignan, France
}

(Published version: Physics of Fluids 31, 065113 (2019); https://doi.org/10.1063/1.5098389)

\begin{abstract}
This paper studies the large-eddy simulation of anisothermal low Mach number turbulent channel flows. We consider the large-eddy simulations of the low Mach number equations in two formulations, the Velocity formulation and the Favre formulation. In both formulations, we investigate the subgrid-scale modelling of the two most significant subgrid terms of the filtered low Mach number equations: the momentum convection subgrid term and the density-velocity correlation subgrid term. To this end, the predictions of large-eddy simulations implementing the models are compared to filtered direct numerical simulations. We address several types of subgrid-scale models: functional eddy-viscosity or eddy-diffusivity models, structural models, tensorial models, and dynamic versions of these models. For the momentum convection subgrid term, we recommend the use of the scale-similarity model and the constant-parameter or dynamic tensorial anisotropic minimum-dissipation (AMD) model. For the densityvelocity correlation subgrid term, several models are able to improve temperaturerelated statistics, for instance the AMD model and the scale-similarity model. More accurate results are obtained with the Favre formulation than with the Velocity formulation.
\end{abstract}

\section{Introduction}

Large-eddy simulation (LES) is a family of methods aiming to predict the time-dependent three-dimensional fields of large-scale motions in turbulent flows. Large-scale motions are computed from the resolution of a set of partial differential equations. Small-scale motions, more computationally expensive to resolve, are accounted for by their modelled effect on larger scales. The scale separation may be formalised using a low-pass filter to remove small scales. In this formalism, subgrid-scale models must be involved to close the governing equations of the filtered fields. The analysis depends on the physical and numerical configuration of the simulation. For instance, the application of large-eddy simulation to Rayleigh-Taylor instabilities is reviewed in [70, 71]. Anisothermal wall-bounded flows are found in many engineering applications, such as heat exchangers, combustion chambers or propulsion systems. In some applications, as in concentrated solar power plants [55, 9], the effects of the variations of the fluid properties with temperature should be taken into account. This paper focuses on the large-eddy simulation of anisothermal wall-bounded flows at low Mach number.

\footnotetext{
${ }^{*}$ Corresponding author : adrien.toutant@univ-perp.fr
} 
Wall-bounded turbulent flows are challenging for large-eddy simulation because of the intrinsically anisotropic and inhomogeneous dynamics of near-wall turbulence which typically requires an anisotropic filter. The subgrid-scale modelling should be able to preserve the driving mechanisms of turbulence near the walls [10, 29]. Energy transfers occur both spatially and in between scales $[37,28,14]$ adding additional effects to the energy cascade model from the classical theoretical framework of large-eddy simulation in homogeneous isotropic turbulence [52]. Besides, the suitability of the asymptotic near-wall behaviour of the subgrid-scale models is crucial to avoid excessive dissipation levels in the near-wall region $[42,43]$. The large-eddy simulation of an isothermal fully-developed channel flow was studied by numerous authors. Related to our study are the simulations at moderate friction Reynolds numbers: 180-220 [60, 1, 25, 30, 51, 45, 24, 69], 360-395 [39, 25, 66, 26, 6, 49, 64] and $556-590[60,30,38,6,13,65,31,51,50,27,57]$.

In strongly anisothermal wall-bounded flows, large-eddy simulations should in addition be able to represent accurately the interaction between temperature and turbulence. Large variations of density, viscosity and thermal conductivity are produced by a temperature gradient. This leads to a strong temperature-velocity coupling, notwithstanding the compressibility effects due to velocity being negligible. Analyses of strongly anisothermal channel flows have been carried out using large-eddy simulations $[11,34,35,54,55,56$, $53,47,48,63,8]$ and direct numerical simulations (DNS) [44, 41, 62, 3, 4, 17, 19, 21]. As the velocity of the fluid is low compared to the speed of sound, the low Mach number equations may be used to relieve the numerical constraints related to acoustic waves. The filtering of the low Mach number equations in the context of large-eddy simulation has been investigated by Dupuy et al. [18], which identified two formulations: the Velocity formulation and the Favre formulation. The two formulations give rise to specific subgrid terms. However, the two most significant subgrid terms are in both cases related to the momentum convection and correlation between density and velocity. The effect of these subgrid terms is crucial for the prediction of the turbulent fields by large-eddy simulations. An a priori study of their modelling was conducted by Dupuy et al. [20] using only the results of direct numerical simulations. However, large-eddy simulations implementing the models are necessary to assess the consequence of the modelling and numerical errors for the predictive capability of the models in a simulation.

This paper investigates a posteriori the modelling of the momentum convection subgrid term and the density-velocity correlation subgrid term in anisothermal turbulent channel flows. It follows up on a previous paper focused on incompressible isothermal channel flows [22]. The results are extended both in regard to the importance of the coupling between temperature and turbulence for the subgrid-scale modelling and to the modelling of the additional subgrid term related to the correlation between density and velocity. Large-eddy simulations of fully developed anisothermal channel flows are performed at two friction Reynolds number, $R e_{\tau}=180$ and $R e_{\tau}=395$. In order to facilitate the interpretation of the results, the large-eddy simulations are compared to direct numerical simulations of the same geometry $[19,21]$ filtered at the resolution of the large-eddy simulations. The analysis relies on the LES formalism introduced by Leonard [33], in which large-eddy simulations aim to provide resolved fields whose statistics correspond to the statistics of a filtered direct numerical simulation. Following Dupuy et al. [22], we address the effect of functional eddyviscosity or eddy-diffusivity models, structural models, tensorial eddy-viscosity models and dynamic versions of these models. We verify the applicability of the models for the subgrid term associated with momentum convection and analyse the modelling of the densityvelocity correlation subgrid term in the Velocity and Favre formulations. The accuracy of the large-eddy simulation is determined by the physical relevance of the subgrid-scale 
models with regard to the filtering operation and the numerical method. The robustness of the models to variations of the grid resolution and of the Reynolds numbers is considered.

We give the resolved equations in section 2 and the subgrid-scale models investigated in section 3. The channel flow configuration and the numerical method are presented in section 4 . The results are discussed in section 5 .

\section{Filtered low Mach number equations}

We consider the large-eddy simulation of the low Mach number equations in two formulations as introduced in Dupuy et al. [18]. The Velocity formulation expresses the filtered low Mach number equations in terms of variables filtered with the unweighted classical filter $(-)$. The Favre formulation expresses the filtered low Mach number equations using Favre-filtered variables, that is based on the density-weighted Favre filter $(\widetilde{r})$ defined for any field $\psi$ as $\widetilde{\psi}=\overline{\rho \psi} / \bar{\rho}$. The two formulations involve a different set of subgrid terms. However, the two most significant subgrid terms are similar in the two formulations $[15,16,18]$. In both cases, a subgrid term is related to the nonlinearity of momentum convection and another related to the correlation of density and velocity. Excluding all other subgrid terms, the filtered low Mach number equations are given in the Velocity formulation by:

- Mass conservation equation

$$
\frac{\partial \bar{\rho}}{\partial t}+\frac{\partial}{\partial x_{j}}\left(\bar{\rho} \bar{U}_{j}+F_{\rho U_{j}}\right)=0
$$

- Velocity transport equation

$$
\frac{\partial \bar{U}_{i}}{\partial t}=-\frac{\partial\left(\bar{U}_{j} \bar{U}_{i}+F_{U_{j} U_{i}}\right)}{\partial x_{j}}+\bar{U}_{i} \frac{\partial \bar{U}_{j}}{\partial x_{j}}-\frac{1}{\bar{\rho}} \frac{\partial \bar{P}}{\partial x_{i}}+\frac{1}{\bar{\rho}} \frac{\partial \Sigma_{i j}(\overline{\boldsymbol{U}}, \bar{T})}{\partial x_{j}},
$$

- Energy conservation equation

$$
\frac{\partial \bar{U}_{j}}{\partial x_{j}}=-\frac{1}{\gamma P_{0}}\left[(\gamma-1) \frac{\partial Q_{j}(\bar{T})}{\partial x_{j}}+\frac{\partial P_{0}}{\partial t}\right],
$$

- Ideal gas law

$$
\bar{T}=\frac{P_{0}}{r \bar{\rho}},
$$

and in the Favre formulation by:

- Mass conservation equation

$$
\frac{\partial \bar{\rho}}{\partial t}+\frac{\partial \bar{\rho} \widetilde{U_{j}}}{\partial x_{j}}=0,
$$

- Momentum conservation equation

$$
\frac{\partial \bar{\rho} \widetilde{U}_{i}}{\partial t}=-\frac{\partial\left(\bar{\rho} \widetilde{U}_{j} \widetilde{U}_{i}+\bar{\rho} G_{U_{j} U_{i}}\right)}{\partial x_{j}}-\frac{\partial \bar{P}}{\partial x_{i}}+\frac{\partial \Sigma_{i j}(\widetilde{\boldsymbol{U}}, \widetilde{T})}{\partial x_{j}},
$$


- Energy conservation equation

$$
\frac{\partial}{\partial x_{j}}\left(\widetilde{U}_{j}+\bar{\rho} G_{U_{j} / \rho}\right)=-\frac{1}{\gamma P_{0}}\left[(\gamma-1) \frac{\partial Q_{j}(\widetilde{T})}{\partial x_{j}}+\frac{\partial P_{0}}{\partial t}\right],
$$

- Ideal gas law

$$
\widetilde{T}=\frac{P_{0}}{\bar{\rho} r},
$$

with $\rho$ the density, $T$ the temperature, $\gamma$ the heat capacity ratio, $r$ the ideal gas specific constant, $t$ the time, $P$ the mechanical pressure, $P_{0}$ the thermodynamical pressure, $U_{i}$ the $i$-th component of velocity and $x_{i}$ the Cartesian coordinate in $i$-th direction. Einstein summation convention is used. The functions $\Sigma_{i j}(\boldsymbol{U}, T)$ and $Q_{j}(T)$ are used to compute the shear-stress tensor and conductive heat flux associated with a given velocity and temperature. We assume a Newtonian fluid and Fourier's law,

$$
\begin{gathered}
\Sigma_{i j}(\boldsymbol{U}, T)=\mu(T)\left(\frac{\partial U_{i}}{\partial x_{j}}+\frac{\partial U_{j}}{\partial x_{i}}\right)-\frac{2}{3} \mu(T) \frac{\partial U_{k}}{\partial x_{k}} \delta_{i j}, \\
Q_{j}(T)=-\lambda(T) \frac{\partial T}{\partial x_{j}},
\end{gathered}
$$

with $\mu(T)$ the dynamic viscosity, $\lambda(T)$ the thermal conductivity and $\delta_{i j}$ the Kronecker delta.

The momentum convection subgrid term is defined as $F_{U_{j} U_{i}}=\overline{U_{j} U_{i}}-\bar{U}_{j} \bar{U}_{i}$ in the Velocity formulation and $G_{U_{j} U_{i}}=\widetilde{U_{j} U_{i}}-\widetilde{U}_{j} \widetilde{U}_{i}$ in the Favre formulation. The density-velocity correlation subgrid term is defined as $F_{\rho U_{j}}=\overline{\rho U_{j}}-\bar{\rho} \bar{U}_{j}$ in the Velocity formulation and $G_{U_{j} / \rho}=\widehat{U_{j} / \rho}-\widetilde{U}_{j} / \bar{\rho}$ in the Favre formulation. The two formulations are related by the relation

$$
\frac{F_{\rho U_{j}}}{\bar{\rho}}=-\bar{\rho} G_{U_{j} / \rho}
$$

The fluid is air. We use Sutherland's law [61] to compute the viscosity,

$$
\mu(T)=\mu_{0}\left(\frac{T}{T_{0}}\right)^{\frac{3}{2}} \frac{T_{0}+S}{T+S}
$$

with $\mu_{0}=1.716 \cdot 10^{-5} \mathrm{~Pa} \mathrm{~s}, S=110.4 \mathrm{~K}$ and $T_{0}=273.15 \mathrm{~K}$. We assume a constant Prandtl number $\operatorname{Pr}=0.76$ and heat capacity at constant pressure $C_{p}=1005 \mathrm{~J} \mathrm{~kg}^{-1} \mathrm{~K}^{-1}$. The conductivity is deduced from the Prandlt number, the heat capacity at constant pressure and the viscosity,

$$
\lambda(T)=\frac{C_{p}}{\operatorname{Pr}} \mu(T) .
$$

The ideal gas specific constant is $r=287 \mathrm{~J} \mathrm{~kg}^{-1} \mathrm{~K}^{-1}$.

\section{Subgrid-scale models}

In order to express the models for the momentum convection subgrid term and the density-velocity correlation subgrid term in the Velocity and Favre formulations, it is useful to introduce the following formalism:

$$
F_{U_{j} U_{i}} \approx \tau_{i j}^{\bmod }(\overline{\boldsymbol{U}}, \overline{\boldsymbol{\Delta}}),
$$




$$
\begin{aligned}
G_{U_{j} U_{i}} & \approx \tau_{i j}^{\bmod }(\tilde{\boldsymbol{U}}, \overline{\boldsymbol{\Delta}}), \\
F_{\rho U_{j}} & \approx \pi_{j}^{\bmod }(\overline{\boldsymbol{U}}, \bar{\rho}, \overline{\boldsymbol{\Delta}}), \\
G_{U_{j} / \rho} & \approx \pi_{j}^{\bmod }(\widetilde{\boldsymbol{U}}, 1 / \bar{\rho}, \overline{\boldsymbol{\Delta}}),
\end{aligned}
$$

where the functions $\tau_{i j}^{\bmod }(\boldsymbol{U}, \overline{\boldsymbol{\Delta}})$ and $\pi_{j}^{\bmod }(\boldsymbol{U}, \phi, \overline{\boldsymbol{\Delta}})$ are model-dependent but do not depend on the formulation. For the momentum convection subgrid term, the same set of subgrid-scale models as described in Dupuy et al. [22] is compared. This includes functional models, structural models and tensorial models. In this section, we briefly present each type of modelling and give the construction of corresponding models for the densityvelocity correlation subgrid term.

The functional eddy-viscosity models investigated are the Smagorinsky model [59], the wall-adapting local eddy-viscosity (WALE) model [42], the Sigma model [43], the anisotropic minimum-dissipation (AMD) model [50], and the Kobayashi model [30]. For each of these models, the momentum convection subgrid term is modelled by analogy with molecular diffusion,

$$
\tau_{i j}^{\bmod }(\boldsymbol{U}, \overline{\boldsymbol{\Delta}})=-2 \nu_{e}^{\bmod }(\boldsymbol{g}, \overline{\boldsymbol{\Delta}}) S_{i j},
$$

where $S_{i j}=\frac{1}{2}\left(g_{i j}+g_{j i}\right)$ is the rate of deformation tensor, $\boldsymbol{g}$ the velocity gradient, defined by $g_{i j}=\partial_{j} U_{i}$, and $\nu_{e}^{\text {mod }}$ the subgrid-scale viscosity, which depends on the model. The expressions of the subgrid-scale viscosity for the investigated models are given in appendix A. Eddy-diffusivity models for the density-velocity correlation subgrid term can be constructed using the constant subgrid-scale Prandtl or Schmidt number assumption,

$$
\pi_{j}^{\bmod }(\boldsymbol{U}, \phi, \overline{\boldsymbol{\Delta}})=-\frac{\nu_{e}^{\bmod }(\boldsymbol{g}, \boldsymbol{d}, \overline{\boldsymbol{\Delta}})}{\operatorname{Pr}_{t}} d_{j}
$$

where $\boldsymbol{d}$ is the scalar gradient, defined by $d_{j}=\partial_{j} \phi$, and $\operatorname{Pr}_{t}$ the subgrid-scale Prandtl or Schmidt number. In addition to the Smagorinsky, WALE, Sigma, AMD and Kobayashi models, we consider the scalar anisotropic minimum-dissipation (SAMD) model [2] for the subgrid-scale diffusivity. Besides, an anisotropic version of the Smagorinsky model [20] is constructed using a scaled velocity gradient $\boldsymbol{g}^{\boldsymbol{a}}$, defined by $g_{i j}^{a}=\Sigma_{j}\left(\bar{\Delta}_{j} / \bar{\Delta}\right) \partial_{j} U_{i}$, and a scaled scalar gradient $\boldsymbol{d}^{a}$, defined by $d_{j}^{a}=\Sigma_{j}\left(\bar{\Delta}_{j} / \bar{\Delta}\right) \partial_{j} \phi$, to involve one filter length scale per direction instead of a single filter length scale. Namely, it is expressed for the two subgrid terms as

$$
\begin{aligned}
\tau_{i j}^{\text {An.mod }}(\boldsymbol{U}, \overline{\boldsymbol{\Delta}}) & =-2 \nu_{e}^{\bmod }\left(\boldsymbol{g}^{\boldsymbol{a}}, \overline{\boldsymbol{\Delta}}\right) S_{i j}^{a}, \\
\pi_{j}^{\text {An.mod }}(\boldsymbol{U}, \phi, \overline{\boldsymbol{\Delta}}) & =-\frac{\nu_{e}^{\bmod }\left(\boldsymbol{g}^{\boldsymbol{a}}, \boldsymbol{d}^{\boldsymbol{a}}, \overline{\boldsymbol{\Delta}}\right)}{P r_{t}} d_{j}^{a},
\end{aligned}
$$

with $S_{i j}^{a}=\frac{1}{2}\left(g_{i j}^{a}+g_{j i}^{a}\right)$ the scaled rate of deformation tensor. Numerically, the divergencerelated part of the deviatoric rate of deformation tensor is neglected to compute eddyviscosity models. In other words, we make the hypothesis

$$
\tau_{i j}^{\bmod }(\boldsymbol{U}, \overline{\boldsymbol{\Delta}})=-2 \nu_{e}^{\bmod }\left(S_{i j}-\frac{1}{3} S_{k k}\right) \approx-2 \nu_{e}^{\bmod } S_{i j} .
$$

which is exact in the incompressible isothermal case and an approximation in the anisothermal case. We verified that this approximation does not affect significantly the results of the large-eddy simulations. 
The structural subgrid-scale models investigated are the gradient model [33], defined as

$$
\begin{aligned}
\tau_{i j}^{\text {Grad. }}(\boldsymbol{U}, \overline{\boldsymbol{\Delta}}) & =\frac{1}{12} C^{\text {Grad. }} G_{i j}(\boldsymbol{U}, \overline{\boldsymbol{\Delta}})=\frac{1}{12} C^{\text {Grad. }} \bar{\Delta}_{k}^{2} g_{i k} g_{j k}, \\
\pi_{j}^{\text {Grad. }}(\boldsymbol{U}, \phi, \overline{\boldsymbol{\Delta}}) & =\frac{1}{12} C^{\text {Grad. }} D_{j}(\boldsymbol{U}, \overline{\boldsymbol{\Delta}})=\frac{1}{12} C^{\text {Grad. }} \bar{\Delta}_{k}^{2} g_{i k} d_{k},
\end{aligned}
$$

and the scale-similarity model [5], defined as

$$
\begin{aligned}
\tau_{i j}^{\text {Simil. }}(\boldsymbol{U}, \overline{\boldsymbol{\Delta}}) & =C^{\text {Simil. }}\left(\widehat{U_{j} U_{i}}-\widehat{U}_{j} \widehat{U}_{i}\right), \\
\pi_{j}^{\text {Simil. }}(\boldsymbol{U}, \phi, \overline{\boldsymbol{\Delta}}) & =C^{\text {Simil. }}\left(\widehat{U_{j} \phi}-\widehat{U}_{j} \widehat{\phi}\right),
\end{aligned}
$$

where $\hat{r}$ is a test filter explicitly computed in the large-eddy simulation. The constants $C^{\text {Grad. }}$ and $C^{\text {Simil. }}$ are introduced to enable the artificial amplification or the reduction of the magnitude of the models.

The tensorial eddy-viscosity models investigated are based on the product of an eddyviscosity model $\tau_{i j}^{\bmod }(\boldsymbol{U}, \overline{\boldsymbol{\Delta}})$ and a constant second-order tensor $H_{i j}^{(k)}$. Namely, they are models of the form

$$
\tau_{i j}^{H^{(k)} \bmod }(\boldsymbol{U}, \overline{\boldsymbol{\Delta}})=H_{i j}^{(k)} \tau_{i j}^{\bmod }(\boldsymbol{U}, \overline{\boldsymbol{\Delta}}),
$$

where no implicit summations over $i$ and $j$ are assumed. This formulation allows a different weighting to the eddy-viscosity assumption for each component of the subgrid-scale model, giving more freedom to take into account the anisotropy of the flow. It can equivalently be rewritten using a tensorial eddy-viscosity $H_{i j}^{(k)} \nu_{e}^{\text {mod }}$. Empirically, interesting results are obtained with the tensor $H_{i j}^{(4)}=\left[\chi_{i j}^{x y} \vee \chi_{i j}^{x z}\right][22]$, where [ $]$ are Iverson brackets, evaluating to 1 if the proposition within bracket is satisfied and 0 otherwise, $\wedge$ the logical conjunction (AND), $\vee$ the logical disjunction (OR) and with the notation $\chi_{i j}^{a b}=(i=a \wedge j=b) \vee(i=b \wedge j=a)$. More explicitly, this corresponds to the matrix

$$
H_{i j}^{(4)}=\left(\begin{array}{lll}
0 & 1 & 1 \\
1 & 0 & 0 \\
1 & 0 & 0
\end{array}\right) \text {. }
$$

The generalisation of this procedure to the density-velocity correlation subgrid term, although possible, has not been investigated in this paper.

Dynamic models based on each type of modelling may be constructed using the same general approach, following Germano et al. [23]. We here give the procedure used for the density-velocity correlation subgrid term, as the generalisation to the momentum convection subgrid term is trivial and may be found in Dupuy et al. [22]. The dynamic model constructed from a constant-parameter model $\pi_{j}^{\bmod }(\overline{\boldsymbol{U}}, \bar{\rho}, \overline{\boldsymbol{\Delta}})$ is of the form

$$
\pi_{j}^{\mathrm{dyn}, \bmod }(\overline{\boldsymbol{U}}, \bar{\rho}, \overline{\boldsymbol{\Delta}})=C^{\mathrm{dyn}} \pi_{j}^{\bmod }(\overline{\boldsymbol{U}}, \bar{\rho}, \overline{\boldsymbol{\Delta}}),
$$

where, following the approach of Lilly [36], the parameter $C^{\mathrm{dyn}}$ is computed to minimise the variance of the residual $E_{j}(\overline{\boldsymbol{U}}, \bar{\rho}, \overline{\boldsymbol{\Delta}})=L_{j}(\overline{\boldsymbol{U}}, \bar{\rho})-C^{\mathrm{dyn}} m_{j}(\overline{\boldsymbol{U}}, \bar{\rho}, \overline{\boldsymbol{\Delta}})$. A different dynamic parameter for each component of the subgrid term may also be used. We refer to this method as the tensorial dynamic method for the momentum convection subgrid term and the vectorial dynamic method for the density-velocity correlation subgrid term. As in the (scalar) dynamic method, the tensorial parameter of the model is computed dynamically to minimise for all $j$ the variance of the residual [1], leading to a model of the form

$$
\pi_{j}^{\mathrm{vec}, \mathrm{dyn}, \bmod }(\overline{\boldsymbol{U}}, \bar{\rho}, \overline{\boldsymbol{\Delta}})=C_{j}^{\mathrm{dyn}} \pi_{j}^{\bmod }(\overline{\boldsymbol{U}}, \bar{\rho}, \overline{\boldsymbol{\Delta}}),
$$




\begin{tabular}{|c|c|c|c|c|}
\hline$R e_{\tau}$ & Name & $\begin{array}{l}\text { Number of grid points } \\
\qquad N_{x} \times N_{y} \times N_{z}\end{array}$ & $\begin{array}{l}\text { Dimension of the domain } \\
\qquad L_{x} \times L_{y} \times L_{z}\end{array}$ & $\begin{array}{l}\text { Cell sizes in wall units } \\
\Delta_{x}^{+} ; \Delta_{y}^{+}(0)-\Delta_{y}^{+}(h) ; \Delta_{z}^{+}\end{array}$ \\
\hline 180 & $48 \mathrm{~B}$ & $48 \times 50 \times 48$ & $4 \pi h \times 2 h \times 2 \pi h$ & $0.50-25 ; 34$ \\
\hline 180 & $36 \mathrm{C}$ & $36 \times 40 \times 36$ & $4 \pi h \times 2 h \times 2 \pi h$ & $2.0-22 ; 45$ \\
\hline 180 & $24 \mathrm{C}$ & $24 \times 28 \times 24$ & $4 \pi h \times 2 h \times 2 \pi h$ & $2.0-35 ; 68$ \\
\hline 180 & DNS & $384 \times 266 \times 384$ & $4 \pi h \times 2 h \times 2 \pi h$ & $0.13-4.2 ; 4.2$ \\
\hline 395 & $96 \mathrm{~B}$ & $96 \times 100 \times 64$ & $4 \pi h \times 2 h \times(4 / 3) \pi h$ & $73 ; \quad 0.50-27 ; 36$ \\
\hline 395 & DNS & $768 \times 512 \times 512$ & $4 \pi h \times 2 h \times(4 / 3) \pi h$ & $0.25-4.1 ; 4.6$ \\
\hline
\end{tabular}

Table 1 - Computational domain and grid spacing of the DNS meshes and the LES meshes used at $R e_{\tau}=180$ and $R e_{\tau}=395$. The cell sizes in wall units are computed using the friction velocity of the direct numerical simulations at the cold side.

where no implicit summation over $j$ is assumed. Notice that the dynamic parameter of equations 29 and 30 are multiplicative constants in front of the entire model. They may thus be interpreted as an amplification or reduction factor with regard to the constantparameter version of the model. For each dynamic procedure, the average $\langle\cdot\rangle$ can be computed as a plane average, that is over the homogeneous directions, or as a global average $[46,68,32,7,58]$, that is over the volume of the channel. The parameter of planeaverage dynamic procedures is a function of time and the wall-normal coordinate. The parameter of global-average dynamic procedures is a function of time.

\section{Numerical study configuration}

\subsection{Channel flow configuration}

We investigate the large-eddy simulation of a fully developed three-dimensional turbulent channel flow submitted to a temperature gradient. We denote $(x)$ the streamwise direction, $(z)$ the spanwise direction and $(y)$ the wall-normal direction (figure 1.). The temperature of the cold wall $(y=0)$ is $T_{1}=293 \mathrm{~K}$ and the temperature of the hot wall $(y=2 h)$ is $T_{2}=586 \mathrm{~K}$. Large-eddy simulations of the channel are carried out at the mean friction Reynolds number $R e_{\tau}=180$ and $R e_{\tau}=395$, where $R e_{\tau}$ is the average of the friction Reynolds number at the cold and hot sides. At $R e_{\tau}=180$, the domain size is $4 \pi h \times 2 h \times 2 \pi h$. At $R e_{\tau}=395$, the domain size is $4 \pi h \times 2 h \times 4 / 3 \pi h$.

\subsection{Numerical settings}

Large-eddy simulations of the channel are performed using a finite difference method in a staggered grid system [40,41] and a third-order Runge-Kutta time scheme [67]. A fourth-order centred scheme is used for momentum convection and a second-order centred scheme is used for momentum diffusion. Direct numerical simulations of the channel have been carried out using the same numerical method by Dupuy et al. [19] at $R e_{\tau}=180$ and Dupuy et al. [21] at $R e_{\tau}=395$. We use three LES meshes at $R e_{\tau}=180$, referred to as "48B", "36C" and " $24 \mathrm{C}$ ", and one LES mesh at $R e_{\tau}=395$, referred to as "96B". The meshes are uniform in the homogeneous directions and follow a hyperbolic tangent law in 


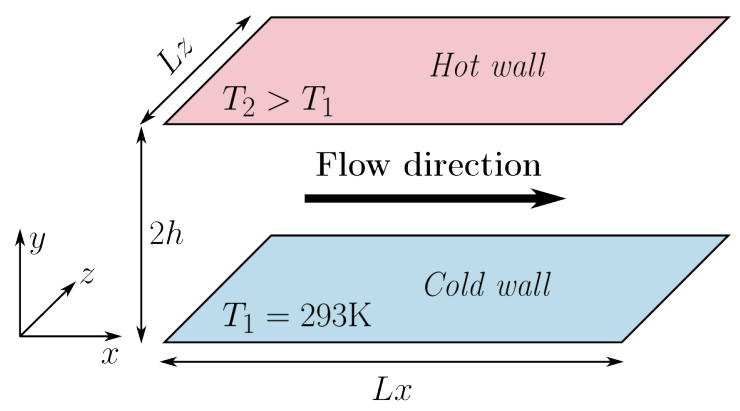

Figure 1 - Biperiodic anisothermal channel flow.

the wall-normal coordinate direction. The number of grid points and the cell sizes of the meshes of the large-eddy simulations and the direct numerical simulations are given in table 1. A streamwise volume force $f$ is added to balance viscous dissipation in the large-eddy simulations and the direct numerical simulations. The same targeted mass flow rate is used in both cases. The resulting wall shear stress may however be different. Accordingly, the mass enclosed in the domain is the same in all simulations but the mean thermodynamical pressure may be different.

\section{3 $\quad$ Filtering process}

The direct numerical simulations are filtered at the resolution of the large-eddy simulations using a top-hat filter. Following Dupuy et al. [18, 20], the box filter is computed using a cubic spline interpolation of the DNS data to allow the convenient use of an arbitrary filter size. We also define two test filters to compute dynamic procedures and the scale-similarity model. "Filter A" is as an average over three cells in the three directions, approximating a top-hat filter with a filter width three times larger than the local cell size. "Filter T", uses the Taylor series expansion of the box filter using the local cell size as the filter width.

\section{Results and discussion}

The large-eddy simulations are used to study the relevance of subgrid-scale models for the momentum convection subgrid term and the density-velocity correlation subgrid term, of combinations of subgrid-scale models for the two subgrid terms and of the use of the Velocity and Favre formulations. Before proceeding to the comparison of the subgrid-scale models, we briefly discuss the simulation of the channel without subgrid-scale model.

To analyse the results, we define the three following scalings:

- The scaling $\left({ }^{+}\right)$, or classical scaling, based on the friction velocity $U_{\tau}$, the wall kinematic viscosity $\nu_{\omega}$ and temperature $T_{\omega}$ and the friction temperature $T_{\tau}$,

$$
y^{+}=\frac{y U_{\tau}}{\nu_{\omega}}, \quad \boldsymbol{U}^{+}=\frac{\boldsymbol{U}}{U_{\tau}}, \quad T^{+}=\frac{T-T_{\omega}}{T_{\tau}} ;
$$

- The scaling $\left(^{\circ}\right)$, based on the channel half-height, the average kinematic viscosity 
$\frac{1}{2}\left(\nu_{1}+\nu_{2}\right)$ and the temperature difference $T_{2}-T_{1}$,

$$
y^{\circ}=\frac{y}{h}, \quad \boldsymbol{U}^{\circ}=\frac{\boldsymbol{U} h}{\frac{1}{2}\left(\nu_{1}+\nu_{2}\right)}, \quad T^{\circ}=\frac{T-T_{\omega}}{T_{2}-T_{1}}
$$

- The scaling $\left(^{\times}\right)$, based on the average friction velocity $\frac{1}{2}\left(U_{\tau, 1}+U_{\tau, 2}\right)$, the average kinematic viscosity $\frac{1}{2}\left(\nu_{1}+\nu_{2}\right)$ and the average friction temperature $\frac{1}{2}\left(T_{\tau, 1}+T_{\tau, 2}\right)$,

$$
y^{\times}=y^{\circ}, \quad \boldsymbol{U}^{\times}=\frac{\boldsymbol{U}}{\frac{1}{2}\left(U_{\tau, 1}+U_{\tau, 2}\right)}, \quad T^{\times}=\frac{T-\frac{1}{2}\left(T_{1}+T_{2}\right)}{\frac{1}{2}\left(T_{\tau, 1}+T_{\tau, 2}\right)} .
$$

In these expressions, $U_{\tau}$ is the friction velocity, defined as $U_{\tau}=\nu_{\omega}\left(\partial_{y}\left\langle U_{x}\right\rangle\right)_{\omega}^{0.5}$ in which the subscript $\omega$ refers to wall values, and $T_{\tau}$ is the friction temperature, defined as $T_{\tau}=$ $\lambda_{\omega}\left(\partial_{y}\langle T\rangle\right)_{\omega} /\left(\rho_{\omega} C_{p} U_{\tau}\right)$

\subsection{Simulation without subgrid-scale models}

The results of the simulations without subgrid-scale model are compared to direct numerical simulations filtered at the resolution of the simulation meshes. The filtering is carried out using the classical filter and the Favre filter. The two filters lead to identical results for the mean streamwise velocity, the mean temperature, the covariance of streamwise and wall-normal velocity and the standard deviation of velocity components and of temperature (figures 2 and 3). The distinction is only relevant for the mean wall-normal velocity, which is different in the Velocity and Favre formulations. The mean filtered wallnormal velocity requires a longer averaging time than the other turbulence statistics and is not well-converged. We may only guess that the mean filtered wall-normal velocity has a slightly lower amplitude than the mean nonfiltered wall-normal velocity with the classical filter and a significantly lower amplitude with the Favre filter. This may be seen in figure 4 , in which the convective heat flux $\left\langle U_{y}\left(\gamma P_{0}\right) /(\gamma-1)\right\rangle$ represents up to a constant the mean wall-normal velocity $\left\langle U_{y}\right\rangle$.

The heat flux at the wall is underestimated by all simulations and by the coarser simulations in particular. This error directly impacts the mean wall-normal velocity. Indeed, without subgrid-scale models, the energy conservation equation leads with the low Mach number hypothesis to a balance between the mean wall-normal velocity and the mean local conductive heat flux,

$$
\frac{\gamma P_{0}}{\gamma-1}\left\langle U_{y}\right\rangle=-\left[\left\langle Q_{y}\right\rangle-Q_{0}\right]
$$

where $Q_{0}$ is the conductive heat flux at $y=0$ (cold wall). Since the heat flux at the center of the channel is well predicted by all simulations, the error on the mean wallnormal velocity is closely related to the error on the wall heat flux (figure 4). The mean wall-normal velocity appears underestimated if it is compared to the classical-filtered DNS data. In all simulations, the mean thermodynamical pressure is larger than in the direct numerical simulation. The error is however not significant compared to the error on the heat flux, and does not exceed $4 \%$ with the mesh $24 \mathrm{C}$ at $R e_{\tau}=180$.

The asymmetry between the profiles of the turbulence statistics at the hot and cold sides is not captured correctly by the simulations. The mean streamwise velocity is overestimated at the cold side and underestimated at the hot side (figures 2(a) and 3(a)). Besides, the amplitude asymmetry between the profiles of the standard deviation of streamwise velocity 
(a)
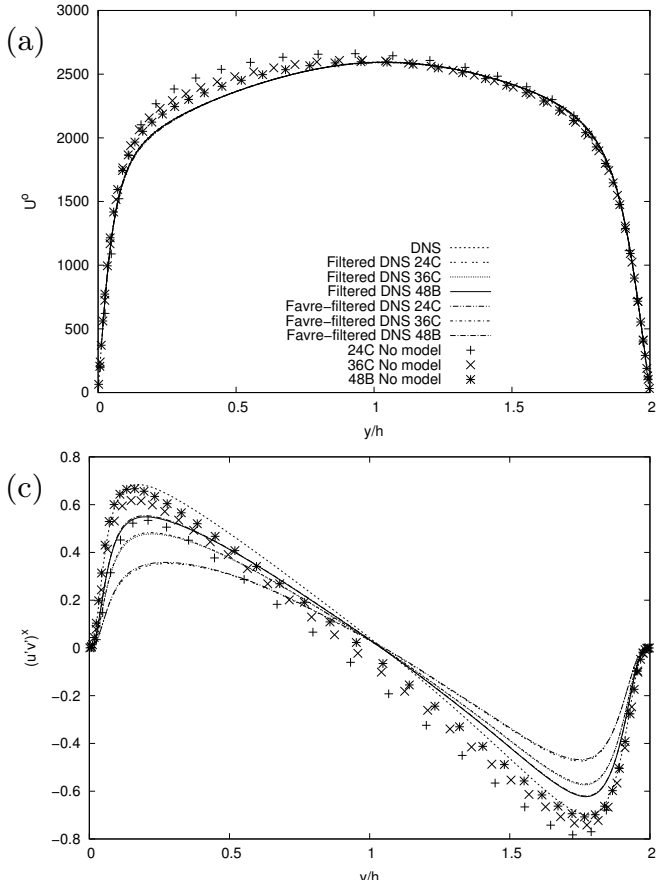

(e)
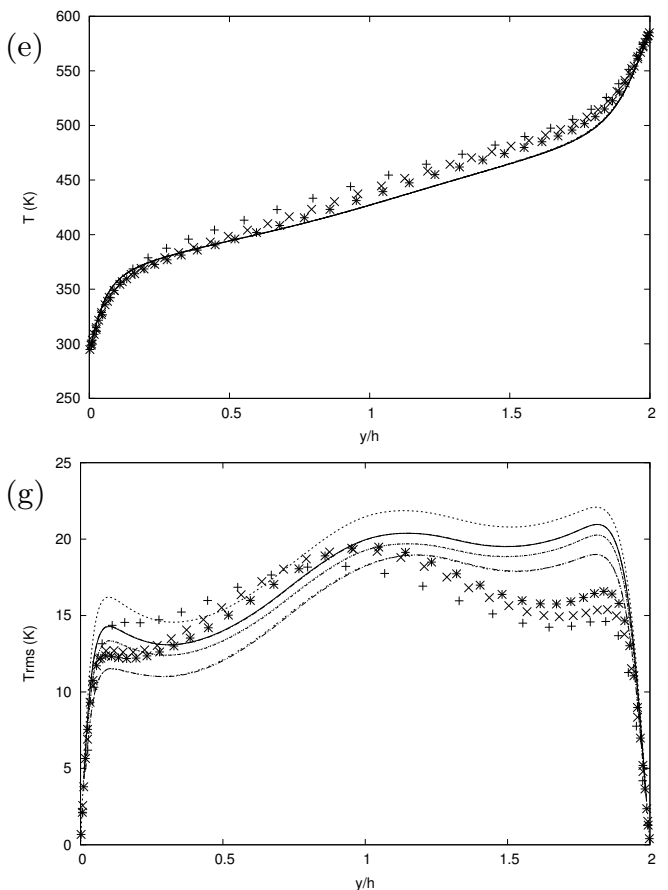

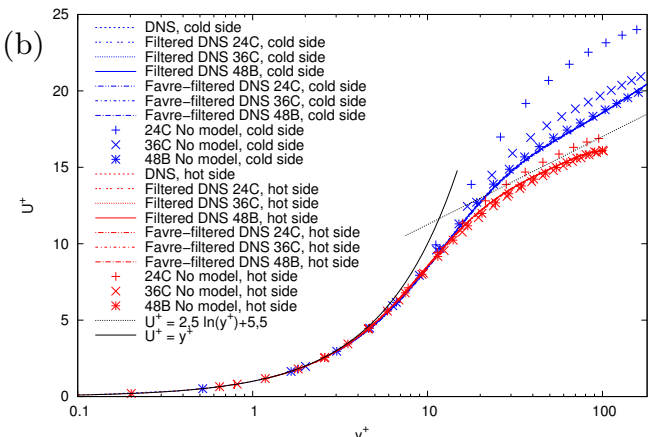

(d)
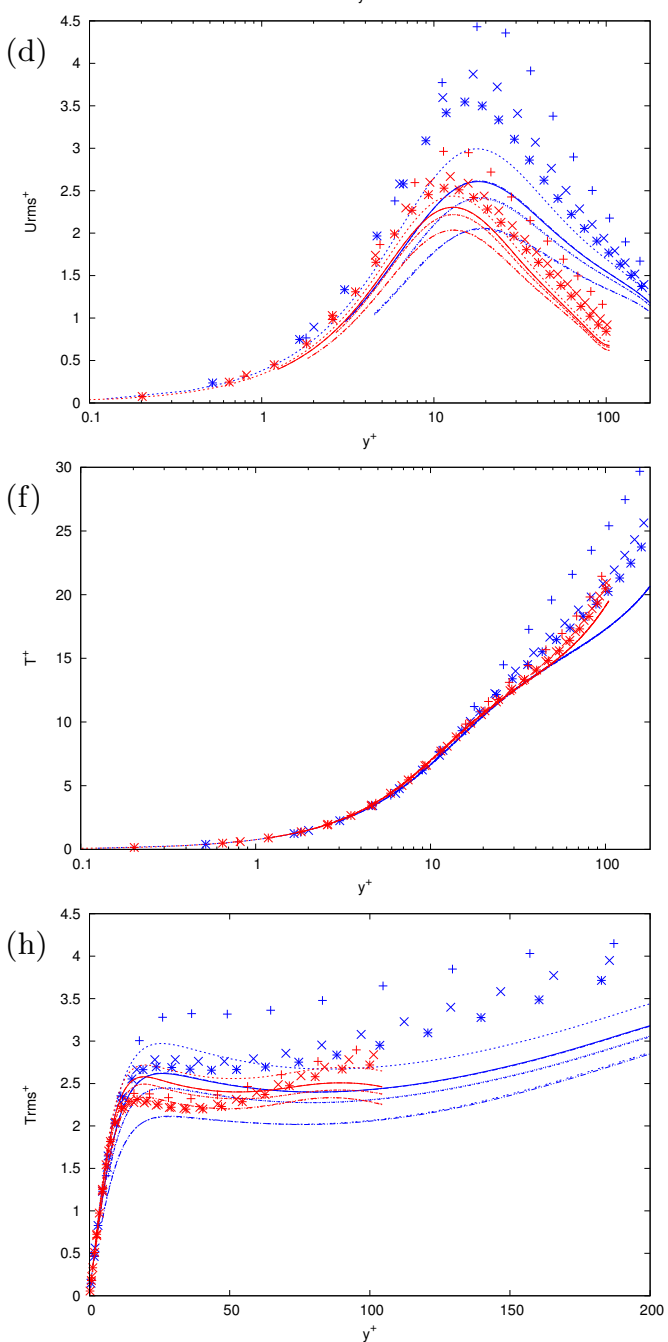

Figure 2 - (Colour online) Comparison of simulations with no subgrid-scale model with the meshes $24 \mathrm{C}, 36 \mathrm{C}$ and $48 \mathrm{~B}$ for the profiles of the mean streamwise velocity $\left\langle U_{x}\right\rangle(\mathrm{a}, \mathrm{b})$, the covariance of streamwise and wall-normal velocity $\left\langle u_{x}^{\prime} u_{y}^{\prime}\right\rangle(\mathrm{c})$, the standard deviation of streamwise velocity $\sqrt{\left\langle u_{x}^{\prime 2}\right\rangle}(\mathrm{d})$, the mean temperature $\langle T\rangle$ (e, f) and the standard deviation of temperature $\sqrt{\left\langle T^{\prime 2}\right\rangle}(\mathrm{g}, \mathrm{h})$ at $R e_{\tau}=180$. 

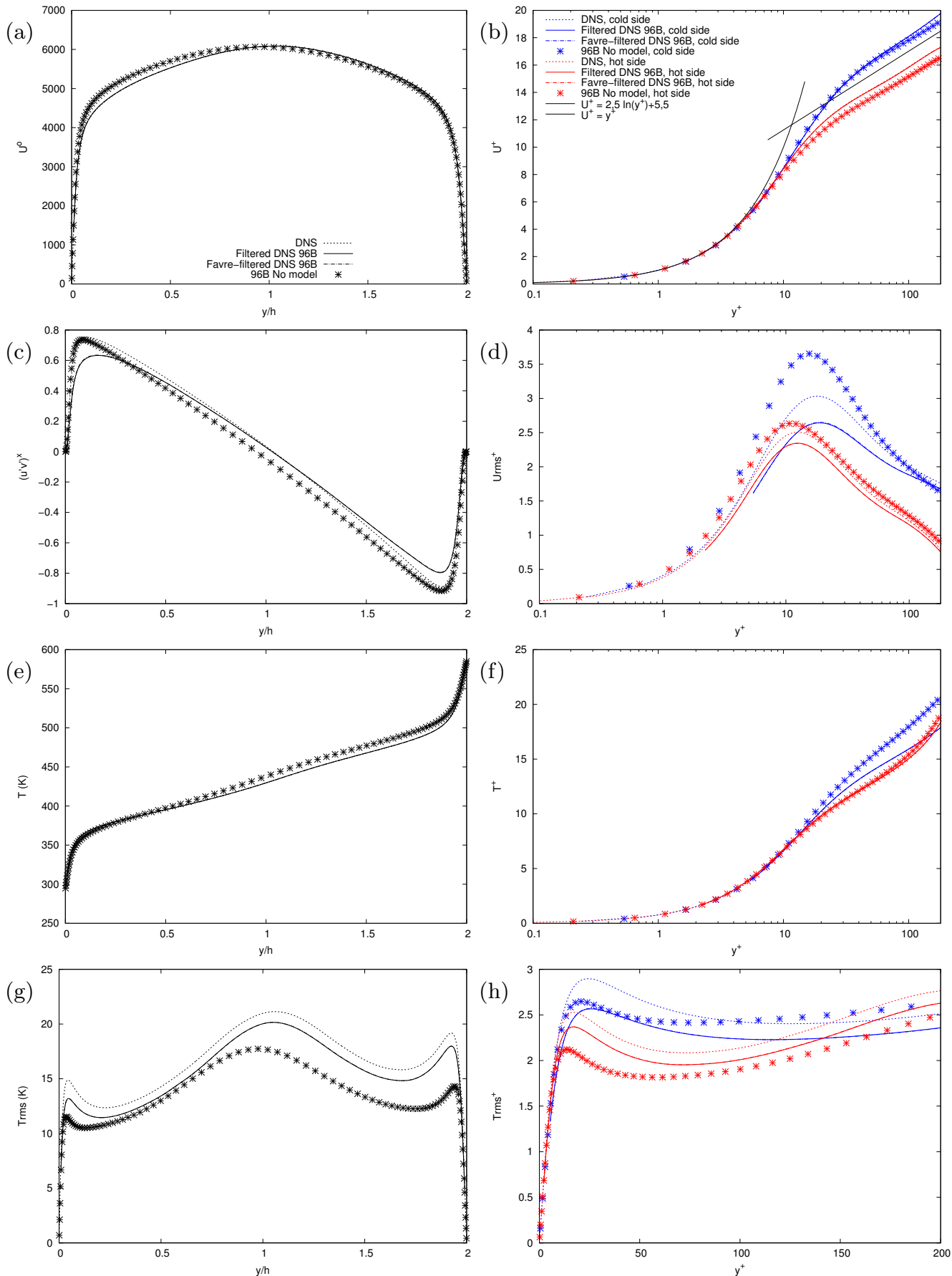

Figure 3 - (Colour online) Comparison of simulations with no subgrid-scale model with the mesh $96 \mathrm{~B}$ for the profiles of the mean streamwise velocity $\left\langle U_{x}\right\rangle(\mathrm{a}, \mathrm{b})$, the covariance of streamwise and wall-normal velocity $\left\langle u_{x}^{\prime} u_{y}^{\prime}\right\rangle$ (c), the standard deviation of streamwise velocity $\sqrt{\left\langle u_{x}^{\prime 2}\right\rangle}$ (d), the mean temperature $\langle T\rangle(\mathrm{e}, \mathrm{f})$ and the standard deviation of temperature $\sqrt{\left\langle T^{\prime 2}\right\rangle}(\mathrm{g}, \mathrm{h})$ at $R e_{\tau}=395$. 

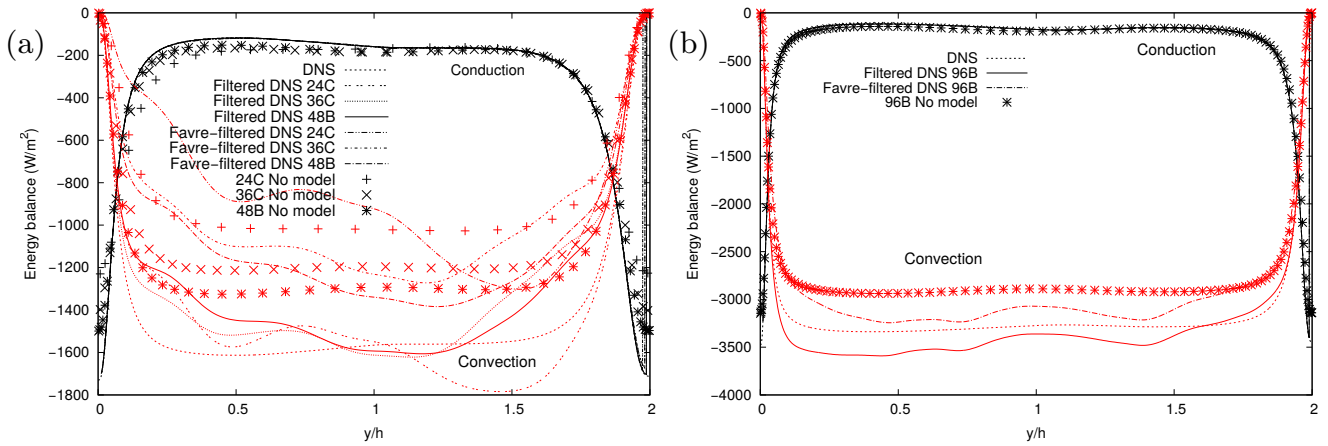

Figure 4 - (Colour online) Comparison of simulations with no subgrid-scale model for the profiles of the terms of the energy balance (31), namely the conductive heat flux $\left\langle U_{y}\left(\gamma P_{0}\right) /(\gamma-1)\right\rangle$ ("Convection") and the conductive heat flux $\langle-\lambda(\partial T / \partial y)\rangle$ ("Conduction") with the meshes $24 \mathrm{C}, 36 \mathrm{C}$ and $48 \mathrm{~B}$ at $R e_{\tau}=180$ (a) and with the mesh $96 \mathrm{~B}$ at $R e_{\tau}=395$ (b).

at the hot and cold sides is amplified compared to the direct numerical simulation (figures $2(\mathrm{c})$ and 3(c)). Conversely, the asymmetry is reduced using the filtered DNS data. This reduction is due to the lower turbulence intensity level at the hot side, leading, in wall units, to an asymmetry of filtering resolution. The mean temperature is without scaling overestimated in the bulk of the channel (figures 2(e) and 3(e)). In other words, the temperature difference to the wall is underestimated at the hot side and overestimated at the cold side. With the classical scaling, it is overestimated at both the hot and cold sides given the error on the wall heat flux (figures 2(f) and 3(f)). For the same reason, the standard deviation of temperature decreases without scaling with mesh derefinement but increases at the cold side with mesh derefinement with the classical scaling (figures $2(\mathrm{~g}, \mathrm{~h}$ ) and $3(\mathrm{~g}, \mathrm{~h}))$.

In the following, we will study the simulation of the anisothermal channels with subgridscale models. We first study the modelling of the subgrid term associated with momentum convection. We then analyse the modelling of the density-velocity correlation subgrid term and compare the Velocity and Favre formulations.

\subsection{Modelling of the momentum convection subgrid term}

In this section, we investigate the effect of subgrid-scale models for the momentum convection subgrid term in the Velocity and Favre formulations. The simulations of this section do not model the density-velocity correlation subgrid term. Since the densityvelocity correlation subgrid term is not modelled, the Velocity and Favre formulations are very similar. With the WALE model for instance, the Velocity and Favre formulations lead to almost identical results at $R e_{\tau}=180$ (figure 5) and $R e_{\tau}=395$. Note however that the Favre formulation tends to predict a slightly lower thermodynamical pressure and wall heat flux than the Velocity formulation for a given model.

The asymmetry between the hot and cold sides should correctly be taken into account by the large-eddy simulation. For instance, a good prediction of the mean streamwise velocity requires an accurate description of the asymmetry of the velocity profile. Without model, the streamwise velocity is overestimated at the cold side and underestimated at the hot side. No functional model or structural model is able to rectify this behaviour (figure 6), although some aggravate it. The scaled velocity requires in addition an accurate prediction 
of the wall shear stress. The functional eddy-viscosity models investigated lead to an underestimation of the wall shear stress, which is more accurate without model. As seen in section 5.1, the symmetric filtering of the asymmetric channel leads, in wall units, to an asymmetry of filtering resolution between the hot and cold sides. The maximum amplitude of the standard deviation of streamwise velocity at the cold side should be reduced to correctly handle the excessive amplitude asymmetry found in no-model simulations. This is achieved by the gradient and scale-similarity models at $R e_{\tau}=180$ (figure 6 ) and $R e_{\tau}=$ 395 (figure 10). However, the functional WALE, Sigma and AMD models decrease the maximum value of the standard deviation of wall-normal and spanwise velocity without similar decrease for streamwise velocity (figure 8). Thus, the turbulence anisotropy is not well predicted. A larger decrease of the standard deviation of streamwise velocity at the cold side can be achieved using tensorial eddy-viscosity models, for instance the tensorial AMD model based on the $H^{(4)}$ tensor or the tensorial global-average dynamic AMD model (figure 7). This improvement of the results of the simulation is not obtained using tensorial models based on the Smagorinsky, WALE, Sigma or Kobayashi models and does not occur using a plane-average or global-average scalar dynamic procedures.

Besides, the modelling of the momentum convection subgrid term has an effect on the profile of temperature. Without model, the temperature gradient at the wall is underestimated. With all functional model investigated, including dynamic versions of these models, the subgrid-scale viscosity further decreases the temperature gradient at the wall, deteriorating the prediction of the wall heat flux further away from the filtered DNS profile. Far from the wall on the other hand, functional models improve the temperature profile compared to the no-model simulation, provided that the parameter of the model is not too strong. This holds for the meshes $24 \mathrm{C}, 36 \mathrm{C}$ and $48 \mathrm{~B}$ at $R e_{\tau}=180$ (figure 9) and the mesh $96 \mathrm{~B}$ at $R e_{\tau}=395$ (figure 10). This is also achieved by the plane-average dynamic Smagorinsky model and tensorial eddy-viscosity models. The structural gradient and scale-similarity models do not have a significant effect on the wall heat flux. The misprediction of the wall heat flux directly impacts the mean wall-normal velocity. The structural models investigated decrease the mean wall-normal velocity. The functional models investigated do not have a large effect on the mean wall-normal velocity with the mesh $48 \mathrm{~B}$ at $R e_{\tau}=180$ but lead to a pronounced decrease with the mesh $24 \mathrm{C}$ (figure 9 ). With or without scaling, functional and structural models increase the standard deviation of temperature at the hot and cold sides. An accurate prediction of the temperature profile, if possible with the models investigated, should come from the modelling of the density-velocity correlation subgrid term.

\subsection{Modelling of the density-velocity correlation subgrid term}

In this section, we investigate the effect of subgrid-scale models for the density-velocity correlation subgrid term in the Velocity and Favre formulations. The simulations of this section do not model the momentum convection subgrid term. Modelling the densityvelocity correlation subgrid term primarily affects temperature-related statistics, as the prediction of the wall heat flux, the mean temperature, the mean wall-normal velocity or the standard deviation of temperature. They may have a small effect on the covariance of streamwise and wall-normal velocity or the standard deviation of wall-normal and spanwise velocity but have, in general, a negligible impact on the mean streamwise velocity and the prediction of the wall shear stress. The density-velocity correlation subgrid term does not appear in the same equation in the Velocity and Favre equations. Its modelling has nevertheless a similar effect on the mean temperature and on the standard deviation 
(a)

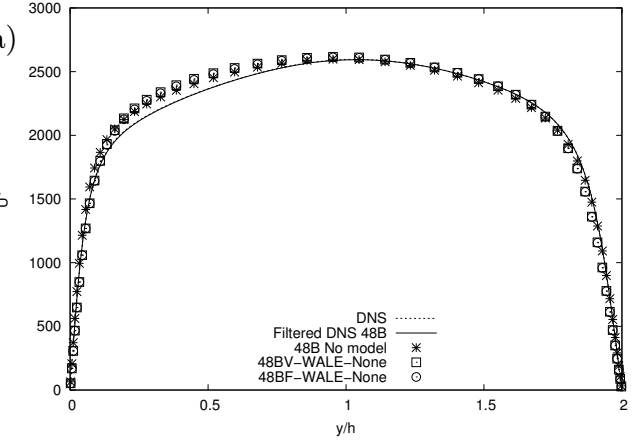

(c)

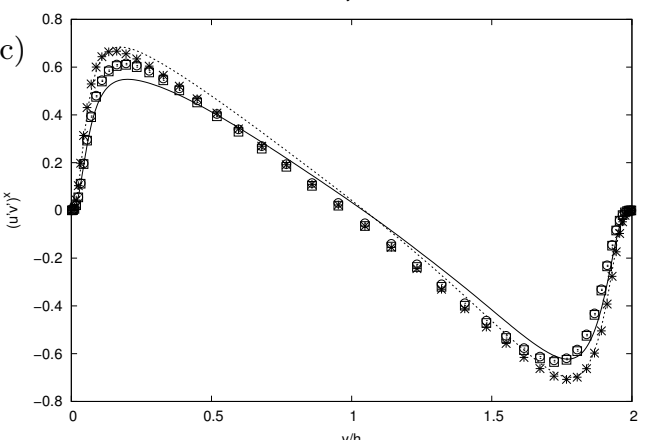

(e)
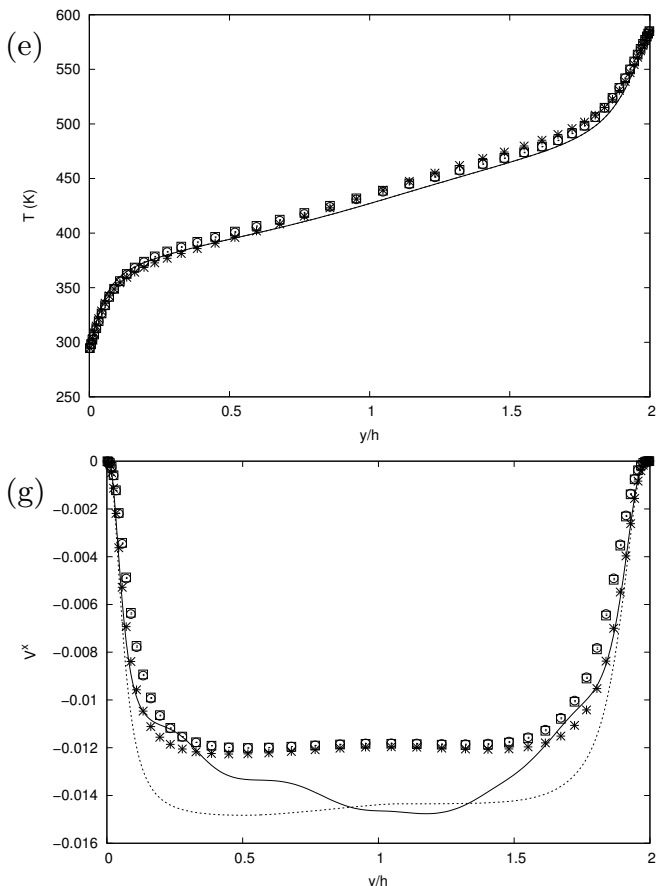

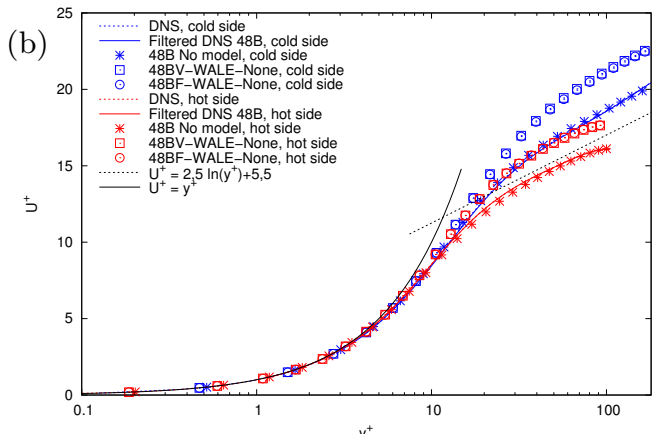

(d)
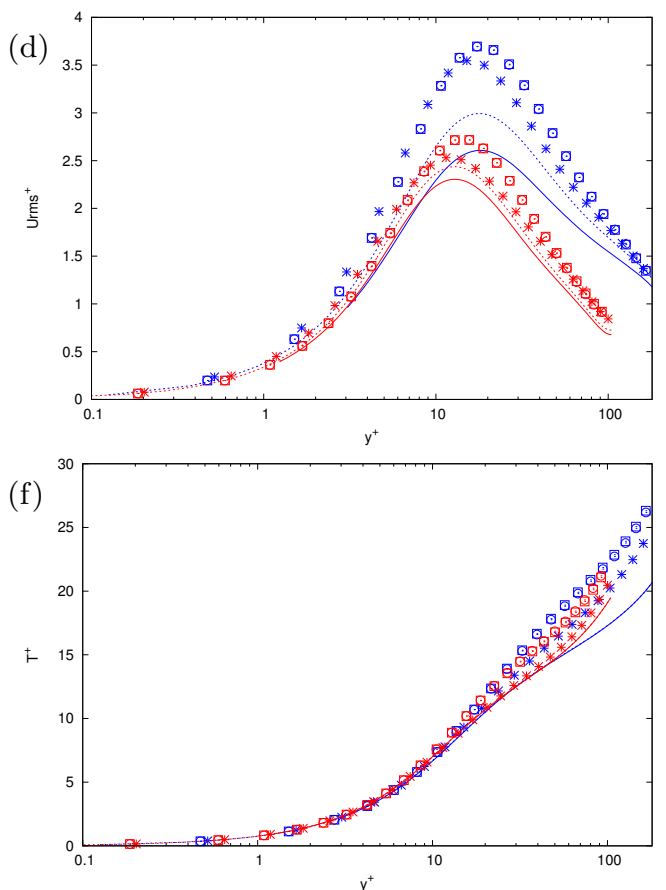

(h)

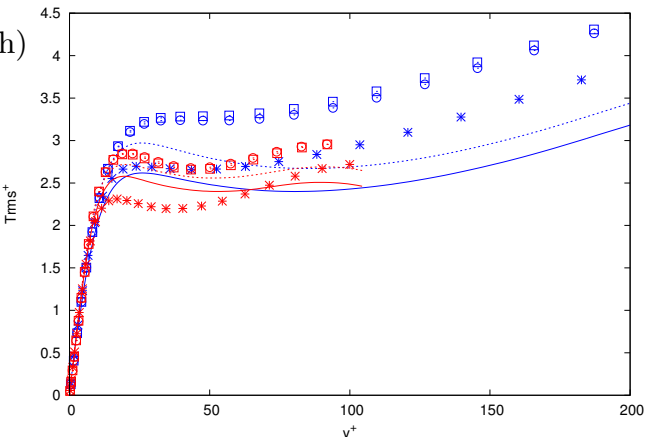

Figure 5 - (Colour online) Comparison of large-eddy simulations with the WALE model for the momentum convection subgrid term in the Velocity and Favre formulations for the profiles of the mean streamwise velocity $\left\langle U_{x}\right\rangle(\mathrm{a}, \mathrm{b})$, the covariance of streamwise and wall-normal velocity $\left\langle u_{x}^{\prime} u_{y}^{\prime}\right\rangle$ (c), the standard deviation of spanwise velocity $\sqrt{\left\langle u_{z}^{\prime 2}\right\rangle}$ (d), the mean temperature $\langle T\rangle$ (e, f), the mean wall-normal velocity $\left\langle U_{y}\right\rangle$ (g) and the standard deviation of temperature $\sqrt{\left\langle T^{\prime 2}\right\rangle}(\mathrm{h})$ at $R e_{\tau}=180$ with the mesh $48 \mathrm{~B}$. 

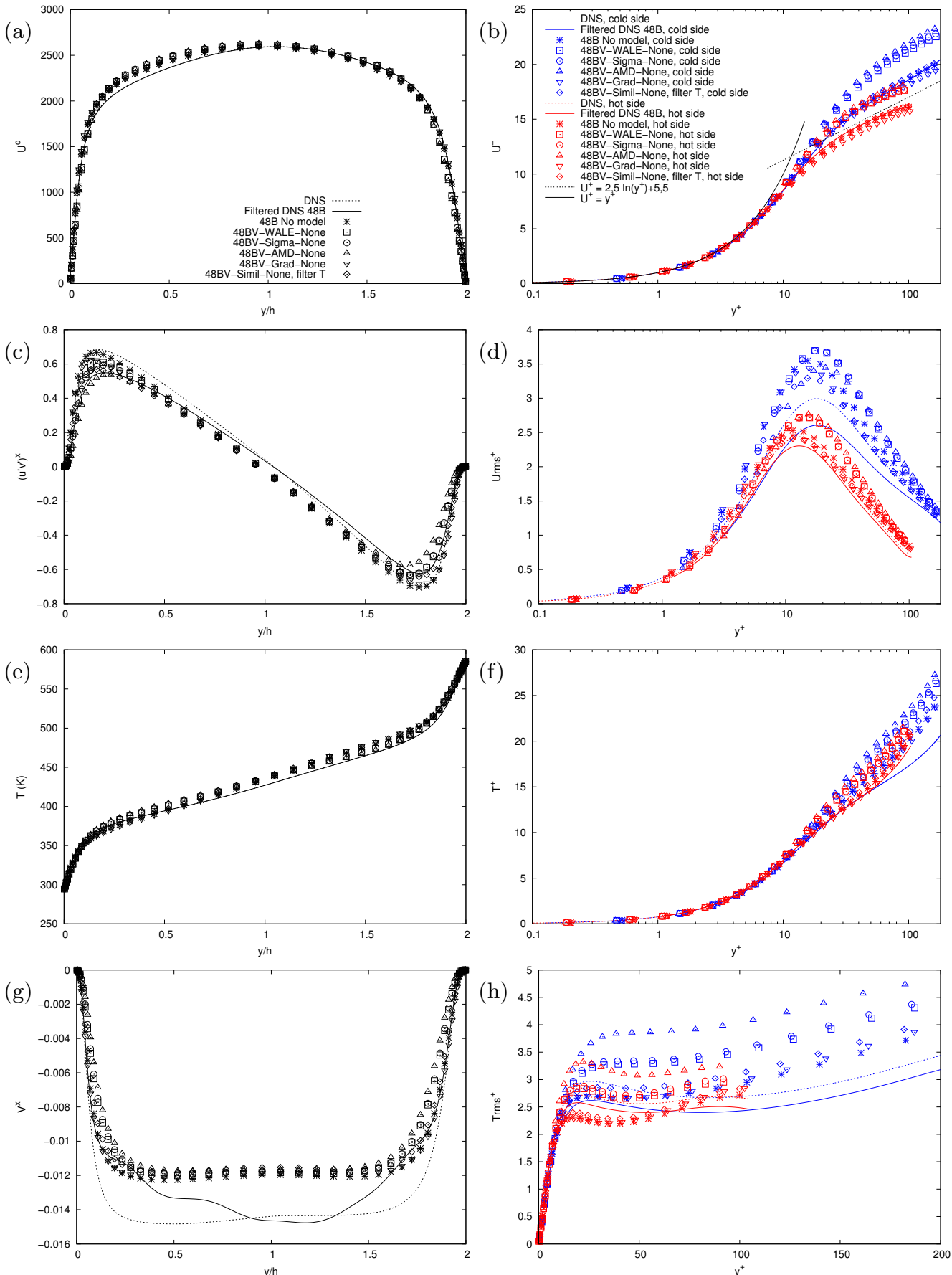

Figure 6 - (Colour online) Comparison of large-eddy simulations with the WALE, Sigma, AMD, gradient (Grad) and scale-similarity (Simil) models for the momentum convection subgrid term in the Velocity formulation for the profiles of the mean streamwise velocity $\left\langle U_{x}\right\rangle(\mathrm{a}, \mathrm{b})$, the covariance of streamwise and wall-normal velocity $\left\langle u_{x}^{\prime} u_{y}^{\prime}\right\rangle$ (c), the standard deviation of spanwise velocity $\sqrt{\left\langle u_{z}^{\prime 2}\right\rangle}(\mathrm{d})$, the mean temperature $\langle T\rangle$ (e, f), the mean wall-normal velocity $\left\langle U_{y}\right\rangle(\mathrm{g})$ and the standard deviation of temperature $\sqrt{\left\langle T^{\prime 2}\right\rangle}$ (h) at $R e_{\tau}=180$ with the mesh $48 \mathrm{~B}$. 
(a)

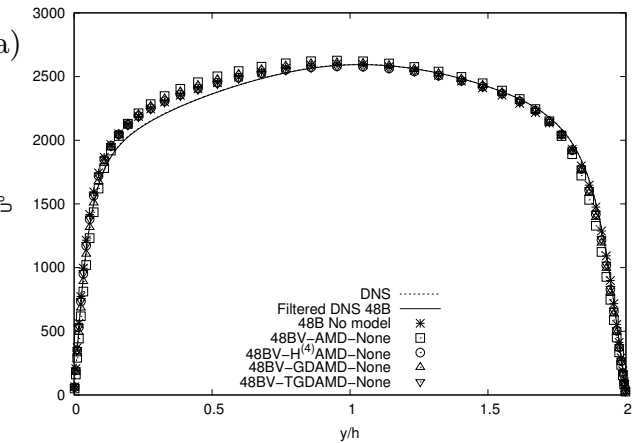

(c)

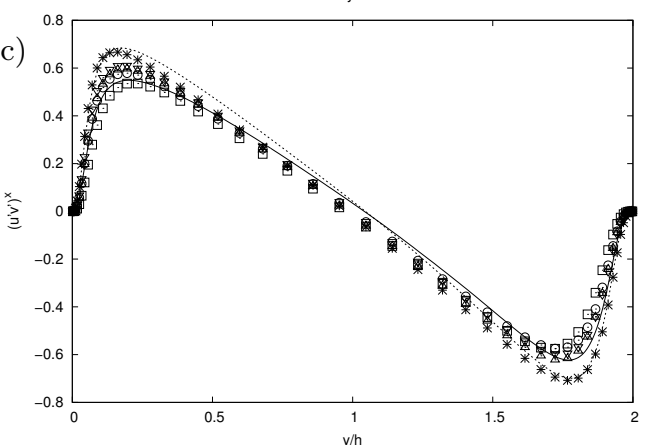

(e)
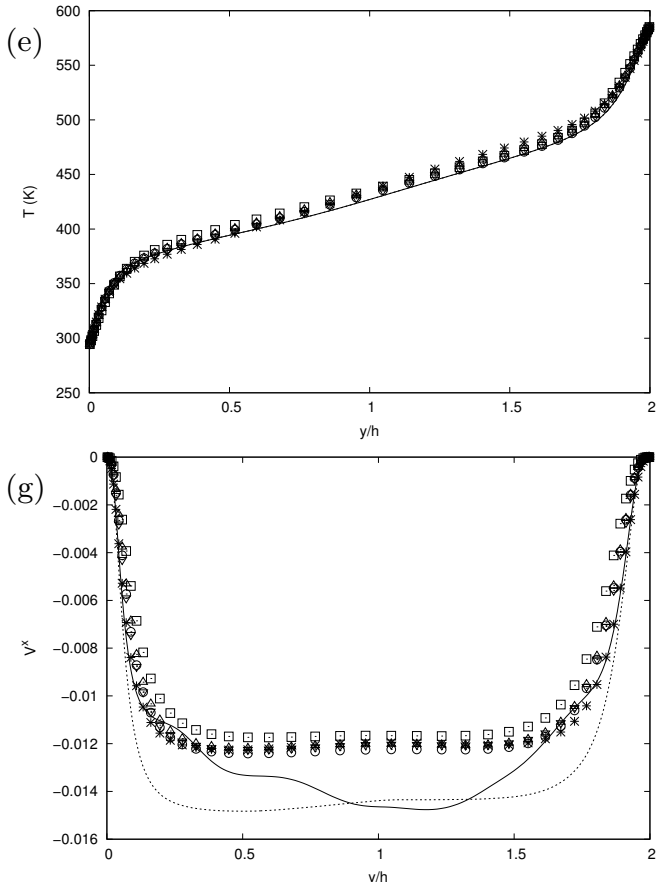

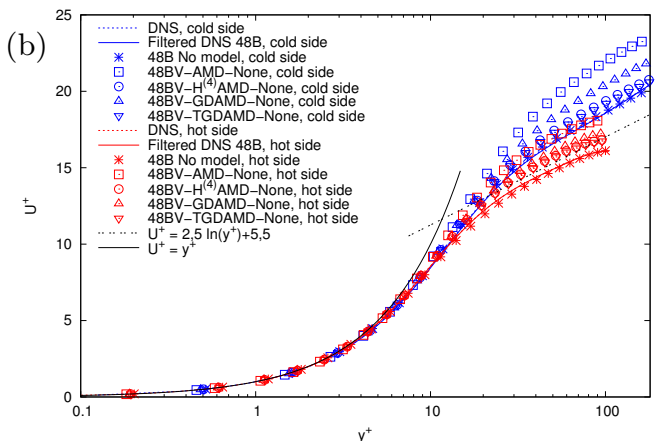

(d)
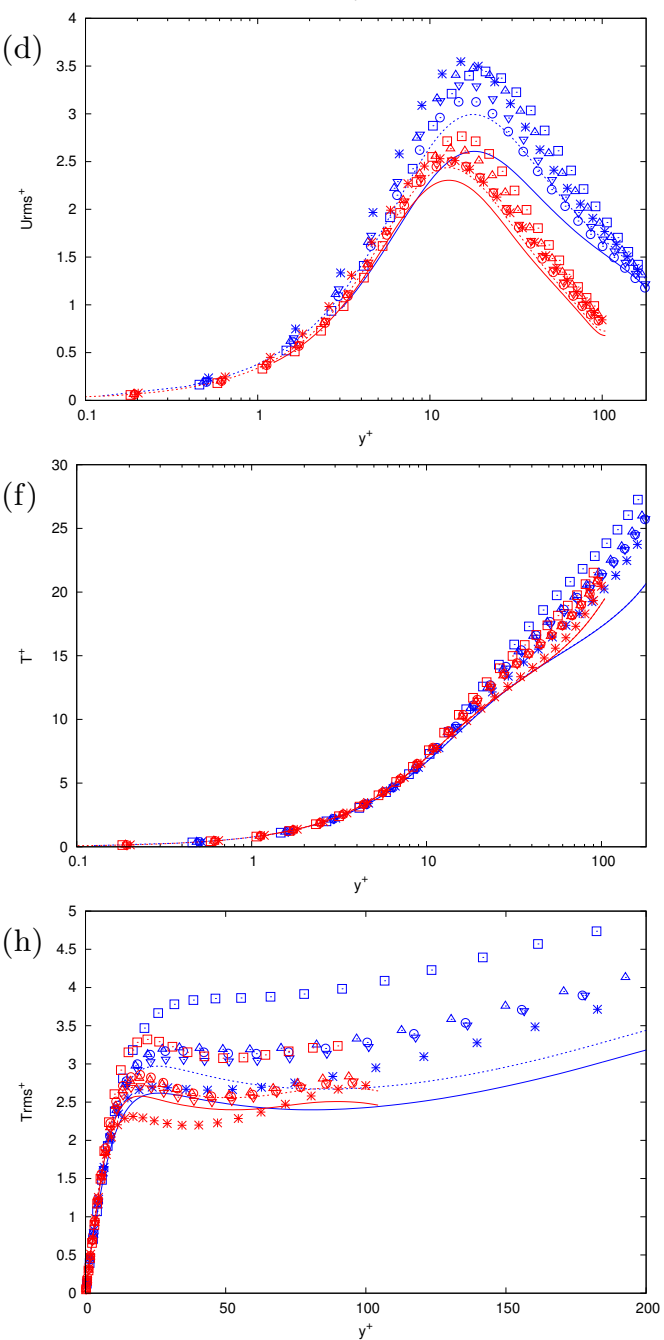

Figure 7 - (Colour online) Comparison of large-eddy simulations with the AMD model, the tensorial AMD model based on the $H^{(4)}$ tensor $\left(H^{(4)} \mathrm{AMD}\right)$ and the global-average (GDAMD) and tensorial global-average (TGDAMD) dynamic AMD models for the momentum convection subgrid term in the Velocity formulation for the profiles of the mean streamwise velocity $\left\langle U_{x}\right\rangle$ (a, b), the covariance of streamwise and wall-normal velocity $\left\langle u_{x}^{\prime} u_{y}^{\prime}\right\rangle$ (c), the standard deviation of spanwise velocity $\sqrt{\left\langle u_{z}^{\prime 2}\right\rangle}(\mathrm{d})$, the mean temperature $\langle T\rangle(\mathrm{e}, \mathrm{f})$, the mean wall-normal velocity $\left\langle U_{y}\right\rangle$ (g) and the standard deviation of temperature $\sqrt{\left\langle T^{\prime 2}\right\rangle}(\mathrm{h})$ at $R e_{\tau}=180$ with the mesh $48 \mathrm{~B}$. 

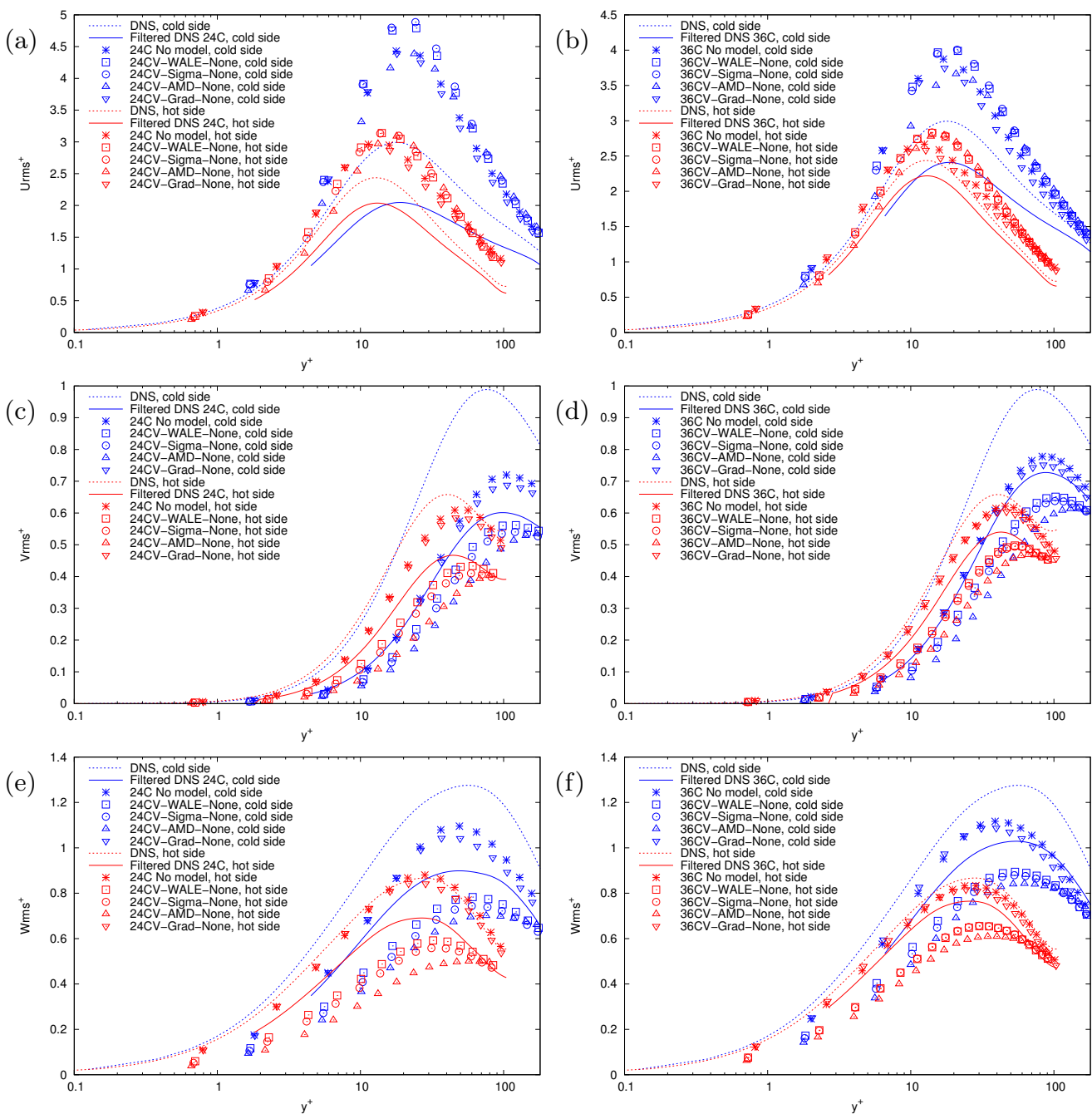

Figure 8 - (Colour online) Comparison of large-eddy simulations with the WALE, Sigma and AMD models for the momentum convection subgrid term in the Velocity formulation with the meshes $24 \mathrm{C}$ (left) and 36C (right) for the profiles of the standard deviation of streamwise velocity $\sqrt{\left\langle u_{x}^{\prime 2}\right\rangle}(\mathrm{a}, \mathrm{b})$, wall-normal velocity $\sqrt{\left\langle u_{y}^{\prime 2}\right\rangle}(\mathrm{c}, \mathrm{d})$ and spanwise velocity $\sqrt{\left\langle u_{z}^{\prime 2}\right\rangle}(\mathrm{e}, \mathrm{f})$ at $\operatorname{Re}_{\tau}=180$.

of temperature in the Velocity and Favre formulations (figure 11). The effect of the formulation is the greatest on the mean wall-normal velocity. This is consistent with the fact that the classical-filtered and Favre-filtered statistics are identical except for the mean wall-normal velocity.

Every model investigated in figures 11-15 increases the wall heat flux compared to the no-model simulation. This improves the results of the simulation since the wall heat flux is underestimated without model. At $R e_{\tau}=180$, the increase is larger with the mesh $24 \mathrm{C}$ than with the mesh $48 \mathrm{~B}$, but not sufficiently. Indeed, the DNS wall heat flux is exceeded with the mesh $48 \mathrm{~B}$ while still not attained with the mesh $24 \mathrm{C}$ using the WALE model with the parameter $C^{\mathrm{WALE}}=0.95$ or the scale-similarity model using filter $\mathrm{A}$. In addition, most models decrease the standard deviation of temperature at the hot and cold sides (figures 11(f)-15(f)). The decrease occurs in the Velocity and Favre formulations but is slightly more pronounced in the Velocity formulation. These findings hold for functional and struc- 

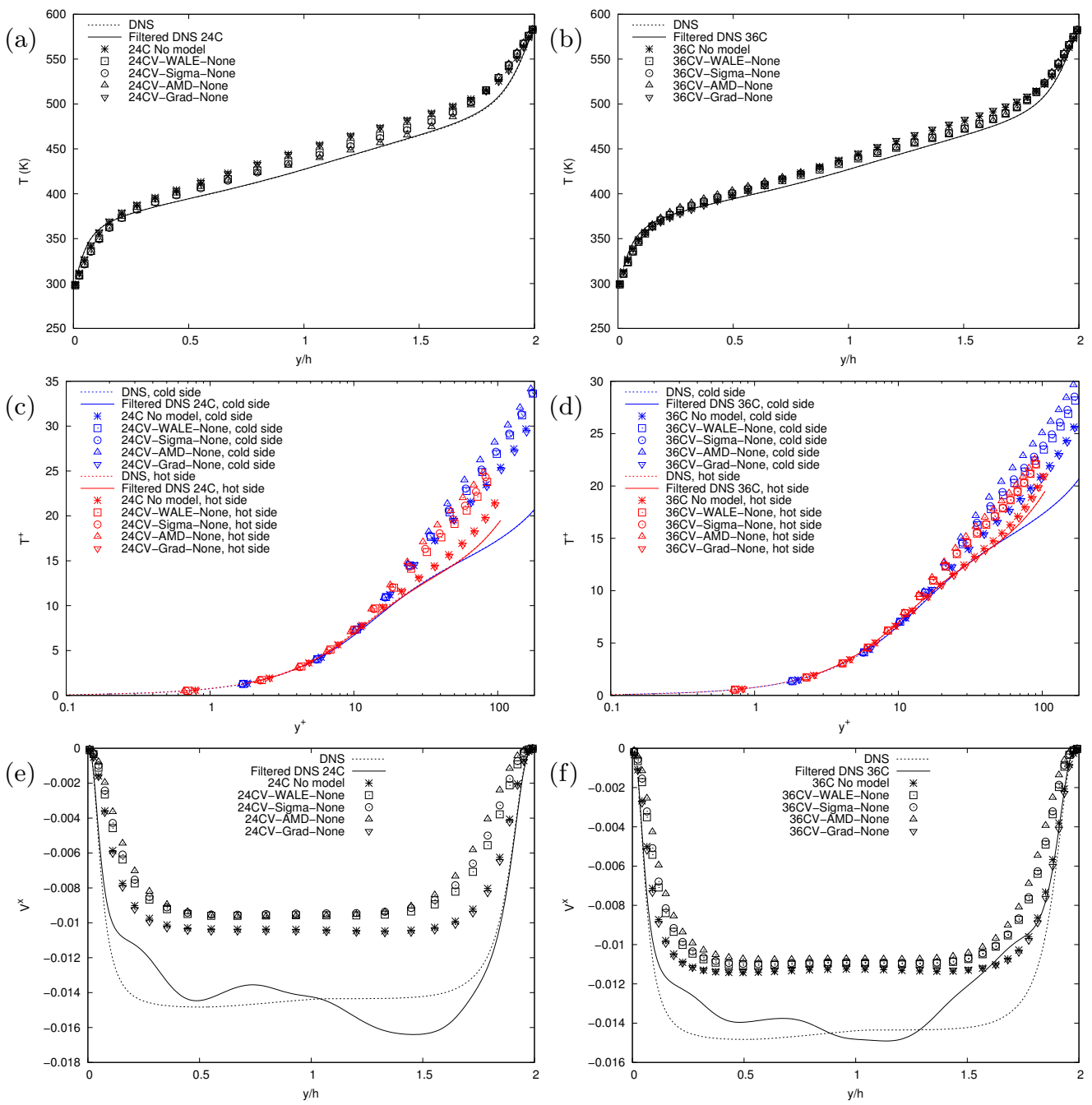

Figure 9 - (Colour online) Comparison of large-eddy simulations with the WALE, Sigma and AMD models for the momentum convection subgrid term in the Velocity formulation with the meshes $24 \mathrm{C}$ (left) and $36 \mathrm{C}$ (right) for the profiles of the mean temperature $\langle T\rangle$ (a, b, c, d) and the mean wall-normal velocity $\left\langle U_{y}\right\rangle(\mathrm{e}, \mathrm{f})$ at $R e_{\tau}=180$.

tural models. However, the effect of the models on turbulence statistics is not very strong at $R e_{\tau}=180$ using the same model parameters as for the momentum convection subgrid term. Larger parameters are required. For functional models, we study parameters corresponding to a subgrid-scale Prandtl or Schmidt number $\operatorname{Pr}_{t}=0.3$. The larger parameter improves the temperature profile with the WALE model (figure 11). The results are identical with the Sigma model. The AMD and scalar AMD models lead to similar results in the Velocity formulation (figure 12). However, they differ in the Favre formulation. In particular, the AMD model increases the mean wall-normal velocity compared to the nomodel simulation while the scalar AMD model leads to a decrease. The scalar AMD model behaves as the other functional models while the AMD model is peculiar. The vectorial global-average dynamic determination of the parameters of functional models decreases the spanwise component of the models and increase the streamwise contribution, compared to the constant-parameter versions (table 2). Similar dynamic parameters are obtained in the Velocity and Favre formulations. This does not modify significantly the effect of the model on the turbulence statistics (figure 13). As functional models, the classical gradient 
(a)

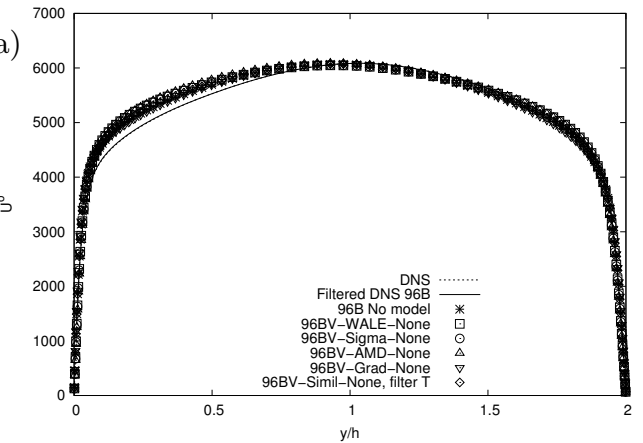

(c)
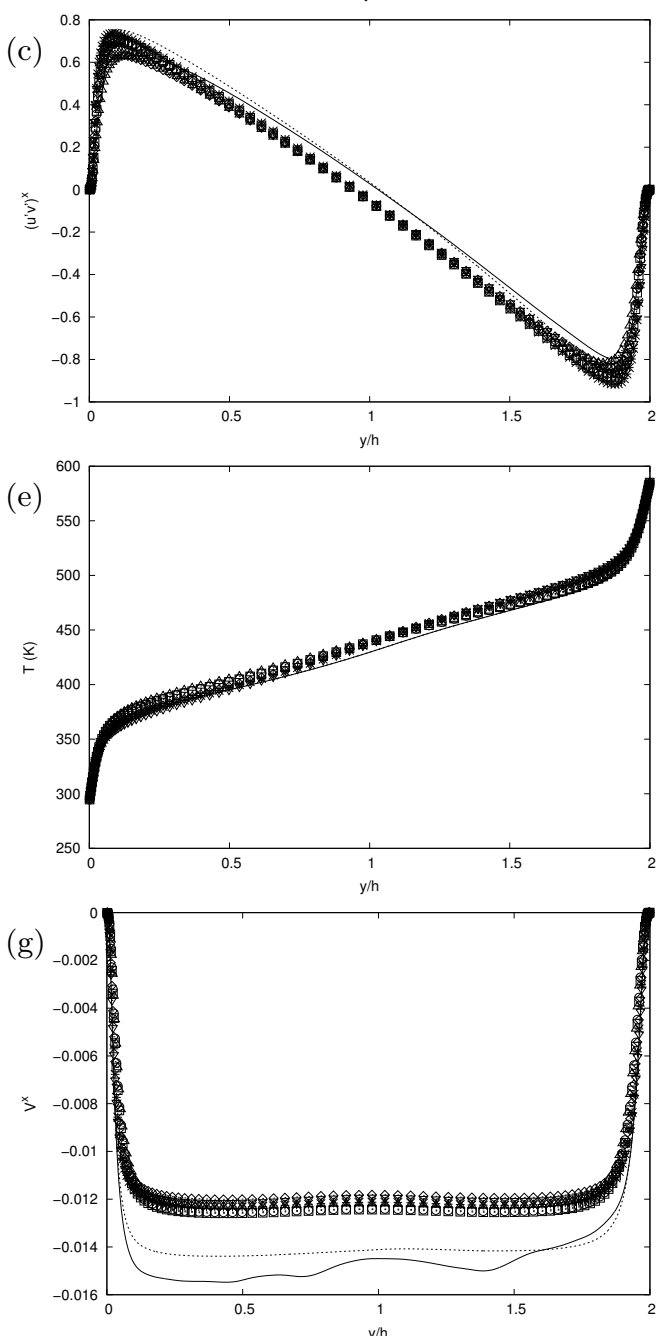

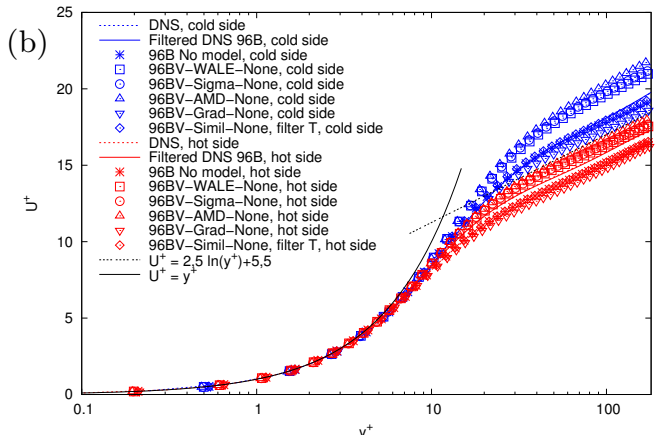

(d)
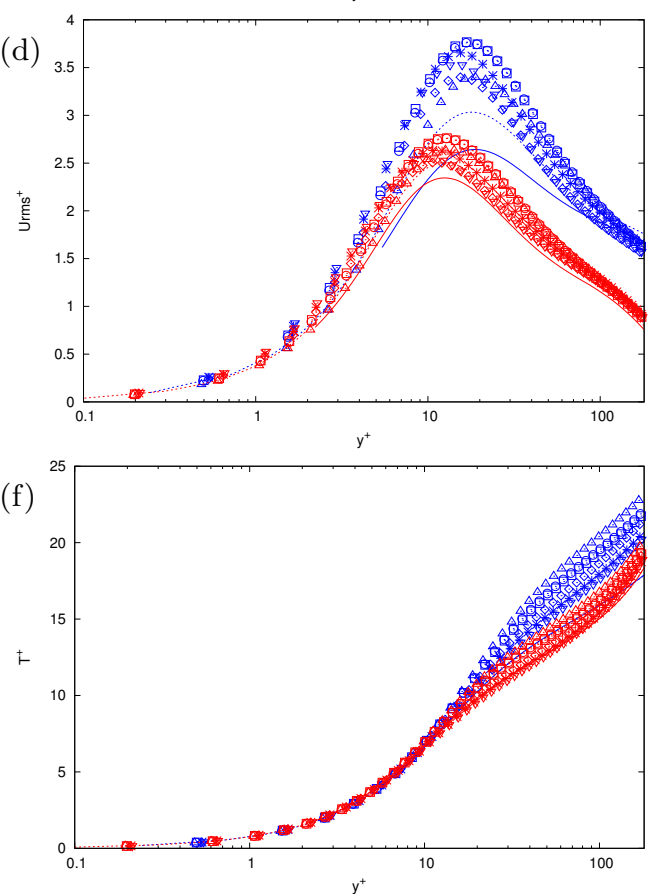

(h)

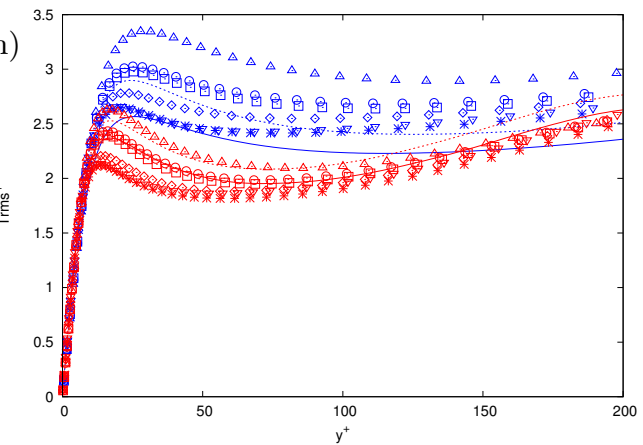

Figure 10 - (Colour online) Comparison of large-eddy simulations with the WALE, Sigma, AMD, gradient (Grad) and scale-similarity (Simil) models for the momentum convection subgrid term in the Velocity formulation for the profiles of the mean streamwise velocity $\left\langle U_{x}\right\rangle(\mathrm{a}, \mathrm{b})$, the covariance of streamwise and wall-normal velocity $\left\langle u_{x}^{\prime} u_{y}^{\prime}\right\rangle$ (c), the standard deviation of spanwise velocity $\sqrt{\left\langle u_{z}^{\prime 2}\right\rangle}(\mathrm{d})$, the mean temperature $\langle T\rangle$ (e, f), the mean wall-normal velocity $\left\langle U_{y}\right\rangle(\mathrm{g})$ and the standard deviation of temperature $\sqrt{\left\langle T^{\prime 2}\right\rangle}$ (h) at $R e_{\tau}=395$ with the mesh 96B. 
Average of the dynamic parameter (standard deviation),

$$
\left\langle C^{\text {mod }}\right\rangle\left(\sqrt{\left\langle\left(C^{\text {mod }}\right)^{2}\right\rangle-\left\langle C^{\text {mod }}\right\rangle^{2}}\right)
$$

Velocity formulation

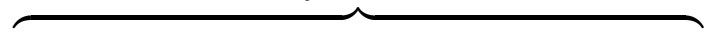

$x$

$y$

$z$

Favre formulation

$\overbrace{y} \quad \overbrace{y}$

\begin{tabular}{lrlllll}
\hline Smag. & $12.976(0.841)$ & $0.021(0.001)$ & $0.184(0.061)$ & $13.405(0.871)$ & $0.005(0.000)$ & $0.165(0.065)$ \\
WALE & $-0.737(0.230)$ & $0.609(0.028)$ & $0.165(0.026)$ & $-0.785(0.289)$ & $0.606(0.029)$ & $0.167(0.027)$ \\
Sigma & $2.348(0.413)$ & $0.840(0.028)$ & $0.372(0.050)$ & $2.415(0.429)$ & $0.834(0.030)$ & $0.366(0.050)$ \\
AMD & $2.101(0.200)$ & $0.356(0.018)$ & $0.167(0.031)$ & $2.237(0.232)$ & $0.360(0.019)$ & $0.156(0.031)$ \\
SAMD & $1.571(0.262)$ & $0.563(0.019)$ & $0.226(0.029)$ & $1.692(0.281)$ & $0.549(0.020)$ & $0.220(0.031)$ \\
Kobayashi & $5.067(0.433)$ & $0.747(0.037)$ & $0.565(0.070)$ & $5.125(0.452)$ & $0.752(0.037)$ & $0.561(0.071)$ \\
An. Smag. & $8.233(0.671)$ & $2.609(0.126)$ & $0.462(0.119)$ & $8.585(0.729)$ & $2.548(0.158)$ & $0.396(0.129)$
\end{tabular}

Table 2 - Average and normalised standard deviation of the dynamic parameters of the large-eddy simulations with the vectorial global-average dynamic Smagorinsky (Smag.), WALE, Sigma, AMD, Scalar AMD (SAMD), Kobayashi and Anisotropic Smagorinsky (An. Smag.) models in the Velocity and Favre formulations at $R e_{\tau}=180$ with the mesh 48B.

model (figure 14) and scale-similarity model (figure 15) have a limited impact on the flow. To amplify the models, we study the scale-similarity model using filter A and the gradient model using $C^{\text {Grad. }}=2$ and using $C^{\text {Grad. }}=9$, filtered to improve the stability of the simulation. We obtain large increase of the wall heat flux and decrease of the standard deviation of temperature. The covariance of streamwise and wall-normal velocity and the standard deviation of wall-normal velocity are also affected. While the amplification of a functional model can be justified using a low subgrid-scale Prandtl or Schmidt number assumption, the amplification of structural models is theoretically not founded.

The above observations suggest that the model for the density-velocity correlation subgrid term is useful and necessary for an accurate prediction of the temperature field. On the other hand, it cannot improve significantly the deficiencies of the section 5.2 regarding statistics not directly related to temperature.

\subsection{Modelling of the momentum convection subgrid term and the density- velocity subgrid term}

In this section, we investigate large-eddy simulation combining models for the momentum convection subgrid term and models for the density-velocity correlation subgrid term. Two sets of simulations are carried out. In the first set of simulations, the two subgrid terms use the same model. In the second set of simulations, the momentum convection subgrid term is the tensorial AMD model based on the $H^{(4)}$ tensor $\left(H^{(4)}\right.$ AMD model) and another model is used for the density-velocity correlation subgrid term.

As suggested by the separate study of the two subgrid terms in section 5.2 and 5.3 , the modelling of the two subgrid terms with a functional model is not appropriate. The WALE model for instance underestimates the wall shear stress and heat flux and overestimates the standard deviation of streamwise velocity and of temperature with the meshes $24 \mathrm{C}, 36 \mathrm{C}$ and $48 \mathrm{~B}$ at $R e_{\tau}=180$ (figure 17 ) or with the mesh $96 \mathrm{~B}$ at $R e_{\tau}=395$. The predictions are in this regard less accurate than with a no-model simulation. These unsatisfactory results 
can mainly be attributed to the functional modelling of the momentum convection subgrid term. The functional modelling of the density-velocity correlation subgrid term decreases the mean temperature and the standard deviation of temperature, bringing the results closer to those of the filtered direct numerical simulation. This holds in the Velocity and Favre formulations. The formulation has a small effect on most turbulence statistics but has a large influence on the mean wall-normal velocity, which is different in the Velocity and Favre formulations. The global-average dynamic procedure provides a less arbitrary determination of the model parameters but the local behaviour of the model is not modified. The plane-average dynamic procedure is only applicable to the Smagorinsky model. The tensorial global-average dynamic procedure is applicable to all functional models investigated and may alter favorably the behaviour of the model. In particular, significant improvements are observed for all turbulence statistics using the tensorial global-average dynamic AMD model for the momentum convection subgrid term and the density-velocity correlation subgrid term compared to the constant-parameter or global-average versions (figure 18).

The $H^{(4)}$ AMD model is one of the most satisfactory models for the momentum convection subgrid term. This model is combined with several models for the density-velocity correlation subgrid term. As expected, the modelling of the density-velocity correlation subgrid term increases the wall heat flux and reduces the standard deviation of temperature. The effect of the model is lower than in simulation without model for the momentum convection subgrid term. At $R e_{\tau}=180$, the $H^{(4)}$ AMD model decreases the prediction of the wall heat flux compared to the no-model simulation. A strong increase from the densityvelocity correlation subgrid term is thus required. This may for instance be achieved with the AMD or scalar AMD model using $C^{\mathrm{SAMD}}=0.90$ or with the scale-similarity model using filter A. With these two models, the wall heat flux and the mean wall-normal velocity is in the Velocity formulation increased significantly up to the level of the direct numerical simulation with the meshes 48B (figure 19) and 36C (figure 20). With the mesh $24 \mathrm{C}$ (figure 21), the effect of the models is no longer sufficient to obtain an accurate prediction of the wall heat flux. At $R e_{\tau}=395$, the $H^{(4)}$ AMD model increases the prediction of the wall heat flux compared to the no-model simulation. The heat flux increase provided by the AMD or scalar AMD model using $C^{\mathrm{SAMD}}=0.90$ or the scale-similarity model using filter A is now excessive (figure 22). In other words, a lower parameter is preferable at $R e_{\tau}=395$ compared to $R e_{\tau}=180$. This has been repeatedly seen in the literature [see for instance 43, 24]. To address this issue, the subgrid-scale model for the momentum convection subgrid term should be less dependent on the friction Reynolds number. The modelling of the density-velocity correlation subgrid term affects more heavily the temperature profile at the cold side in the Favre formulation than in the Velocity formulation, while the opposite is true at the hot side. Since the scaled profile of temperature is without model more accurate at the hot side than at the cold side for all simulations, the Favre formulation gives a more accurate scaled temperature profile than the Velocity formulation. In addition, the streamwise velocity is less overestimated at the cold side in the Favre formulation than in the Velocity formulation, the profile of the covariance of streamwise and wall-normal velocity is closer to the DNS profile at the center of the channel and the predicted mean thermodynamical pressure less overestimated. In both formulations, the standard deviation of temperature is decreased excessively to a lower level than the filtered DNS profile. The isotemperature suface given in figure 16 show that the filtered direct numerical simulation and the large-eddy simulation both have a much lower number of small scales than the direct numerical simulation. However, the isosurface is clearly less irregular in the large-eddy simulation than in the filtered direct numerical simulation. 
Similar results are obtained with most subgrid-scale models.

All in all, while none of the model combinations investigated is able to properly reproduce the asymmetry between the hot and cold sides, some improvements are achieved compared to the no-model case. For the momentum convection subgrid term, interesting results are obtained with the scale-similarity model and the constant-parameter or dynamic tensorial AMD model. Most models investigated seem suitable for the densityvelocity correlation subgrid term. For instance, the AMD model or the scale-similarity models can be used. We recommend the use of the Favre formulation rather than the Velocity formulation.

\section{Conclusion}

The large-eddy simulation of a turbulent anisothermal channel flow shows the challenge presented by the subgrid-scale modelling of shear flows. In this study, the subgrid-scale modelling is addressed from the filtering of the low Mach number equations in two formulations, the Velocity formulation and the Favre formulation. Only the two most significant subgrid terms are considered, the momentum convection subgrid term and the densityvelocity correlation subgrid term. Within the Reynolds number range investigated, the functional modelling of the momentum convection subgrid term do not properly reproduce the asymmetry between the hot and cold sides. Besides, the heat flux at the wall is underestimated and the turbulence anisotropy is misrepresented because the models do not have the same effect on the standard deviation of streamwise velocity as on the wall-normal and spanwise components. Alternative modelling approaches, such as structural models, tensorial models and tensorial global-average dynamic functional models, can improve the results. However, the effect of these models is not in full accord with the filtered direct numerical simulations and is not amplified as expected upon mesh derefinement. The modelling of the other subgrid terms seems necessary for an accurate prediction of the flow. In particular, the modelling of the density-velocity correlation subgrid term is useful and beneficial for the prediction of temperature-related statistics, although it does not significantly alter the velocity field. It has a smaller impact on the flow than the momentum convection subgrid term. Functional models propose a relevant modelling approach for the density-velocity correlation subgrid term since it influences the turbulence statistics pos-

itively. Structural models are also relevant. A subgrid-scale Prandtl or Schmidt number lower than 0.9 was found advisable in order to intensify sufficiently the effect of the model, especially at low Reynolds number. However, the accurate prediction of the flow requires the agreement of the strength of the model with the effect of the model for the momentum convection subgrid term. The Velocity and Favre formulations give almost identical results if only the momentum convection subgrid term is modelled. If the density-velocity correlation subgrid term is taken into account, the formulations primarily affects the mean wall-normal velocity. The results suggest that the Favre formulation is preferable to the Velocity formulation for an accurate prediction of the mean temperature.

\section{Acknowledgments}

This work was funded by the French Investments for the future ("Investissements d'Avenir") programme managed by the National Agency for Research (ANR) under contract ANR-10-LABX-22-01 (labex SOLSTICE). The authors gratefully acknowledge the 
CEA for the development of the TRUST platform. This work was granted access to the HPC resources of CINES under the allocations 2017-A0022A05099 and 2018-A0042A05099 made by GENCI.

\section{A Expressions of the subgrid-scale viscosity}

This appendix gives the subgrid-scale viscosity for the investigated functional models,

Smagorinsky model [59]:

$$
\nu_{e}^{\text {Smag. }}(\boldsymbol{g}, \overline{\boldsymbol{\Delta}})=\left(C^{\text {Smag. }} \bar{\Delta}\right)^{2}|\boldsymbol{S}|,
$$

Wall-adapting local eddy-viscosity (WALE) model [42]:

$$
\nu_{e}^{\mathrm{WALE}}(\boldsymbol{g}, \overline{\boldsymbol{\Delta}})=\left(C^{\mathrm{WALE}} \bar{\Delta}\right)^{2} \frac{\left(\mathcal{S}_{i j}^{d} \mathcal{S}_{i j}^{d}\right)^{\frac{3}{2}}}{\left(S_{m n} S_{m n}\right)^{\frac{5}{2}}+\left(\mathcal{S}_{m n}^{d} \mathcal{S}_{m n}^{d}\right)^{\frac{5}{4}}}
$$

Sigma model [43]:

$$
\nu_{e}^{\text {Sigma }}(\boldsymbol{g}, \overline{\boldsymbol{\Delta}})=\left(C^{\mathrm{Sigma}} \bar{\Delta}\right)^{2} \frac{\sigma_{3}\left(\sigma_{1}-\sigma_{2}\right)\left(\sigma_{2}-\sigma_{3}\right)}{\sigma_{1}^{2}},
$$

Anisotropic minimum-dissipation (AMD) model [50]:

$$
\nu_{e}^{\mathrm{AMD}}(\boldsymbol{g}, \overline{\boldsymbol{\Delta}})=C^{\mathrm{AMD}} \frac{\max \left(0,-G_{i j} S_{i j}\right)}{g_{m n} g_{m n}},
$$

Scalar anisotropic minimum-dissipation (SAMD) model [2]:

$$
\nu_{e}^{\mathrm{SAMD}}(\boldsymbol{g}, \boldsymbol{d}, \overline{\boldsymbol{\Delta}})=C^{\mathrm{SAMD}} \frac{\max \left(0,-D_{j} d_{j}\right)}{d_{m} d_{m}},
$$

Kobayashi model [30]:

$$
\nu_{e}^{\text {Koba. }}(\boldsymbol{g}, \overline{\boldsymbol{\Delta}})=C^{\text {Koba. }} \cdot \bar{\Delta}^{2}\left|F_{g}\right|^{\frac{3}{2}}\left(1-F_{g}\right)|\boldsymbol{S}|,
$$

where $|\boldsymbol{S}|=\sqrt{2 S_{i j} S_{i j}}$ is a norm of $\boldsymbol{S}, \mathcal{S}_{i j}^{d}=\frac{1}{2}\left(g_{i k} g_{k j}+g_{j k} g_{k i}\right)-\frac{1}{3} g_{k p} g_{p k} \delta_{i j}$ the traceless symmetric part of the squared velocity gradient tensor, $\sigma_{1} \geq \sigma_{2} \geq \sigma_{3}$ the three singular values of $\boldsymbol{g}, G_{i j}=\bar{\Delta}_{k}^{2} g_{i k} g_{j k}$ the gradient model for the subgrid term associated with momentum convection, $\mathrm{II}_{G}=\frac{1}{2}\left(\operatorname{tr}^{2}(G)-\operatorname{tr}\left(G^{2}\right)\right)$ its second invariant, $D_{j}=\bar{\Delta}_{k}^{2} g_{j k} d_{k}$ the gradient model for the density-velocity correlation subgrid term, $R_{i j}=\beta_{i} g_{j j}$ the volumetric strain-stretching, with $\beta=\left(S_{23}, S_{13}, S_{12}\right)$, and $F_{g}=\left(\Omega_{i j} \Omega_{i j}-S_{i j} S_{i j}\right) /\left(\Omega_{m n} \Omega_{m n}+S_{m n} S_{m n}\right)$ the coherent structure function, with $\Omega_{i j}=\frac{1}{2}\left(g_{i j}-g_{j i}\right)$ the spin tensor or rate of rotation tensor. Unless stated otherwise, we implicitly use the model parameters $C^{\text {Smag. }}=0.10$, $C^{\mathrm{WALE}}=0.55, C^{\mathrm{Sigma}}=1.5, C^{\mathrm{AMD}}=0.3$ and $C^{\text {Koba. }}=0.045$ and a subgrid-scale Prandt or Schmidt number $P r_{t}=0.9$. The filter length scale is computed following Deardorff [12] as $\bar{\Delta}=\left(\bar{\Delta}_{x} \bar{\Delta}_{y} \bar{\Delta}_{z}\right)^{1 / 3}$. 


\section{References}

[1] A. Abba, A. C. Cercignani, and L. Valdettaro. Analysis of subgrid scale models. Comput. Math. Appl., 46(4):521-535, 2003.

[2] M. Abkar, H. J. Bae, and P. Moin. Minimum-dissipation scalar transport model for large-eddy simulation of turbulent flows. Physical Review Fluids, 1(4):041701, 2016.

[3] F. Aulery, A. Toutant, F. Bataille, and Y. Zhou. Energy transfer process of anisothermal wall-bounded flows. Physics Letters A, 379(24):1520-1526, 2015.

[4] F. Aulery, D. Dupuy, A. Toutant, F. Bataille, and Y. Zhou. Spectral analysis of turbulence in anisothermal channel flows. Computers \& Fluids, 151:115-131, 2017.

[5] J. Bardina, J. Ferziger, and W. C. Reynolds. Improved subgrid-scale models for large-eddy simulation. In 13th Fluid and Plasma Dynamics Conference, page 1357, 1980.

[6] H. Baya Toda, O. Cabrit, G. Balarac, S. Bose, J. Lee, H. Choi, and F. Nicoud. A subgridscale model based on singular values for les in complex geometries. In Proc. of the Summer Program, pages 193-202, 2010.

[7] H. Baya Toda, K. Truffin, and F. Nicoud. Is the dynamic procedure appropriate for all sgs models. In V European Conference on Computational Fluid Dynamics, ECCOMAS, Lisbon, Portugal, pages 14-17, 2010.

[8] M. Bellec, A. Toutant, and G. Olalde. Large eddy simulations of thermal boundary layer developments in a turbulent channel flow under asymmetrical heating. Computers \& Fluids, 151:159-176, 2017.

[9] J. Capeillère, A. Toutant, G. Olalde, and A. Boubault. Thermomechanical behavior of a plate ceramic solar receiver irradiated by concentrated sunlight. Solar Energy, 110:174-187, 2014.

[10] D. Chung and D. I. Pullin. Large-eddy simulation and wall modelling of turbulent channel flow. Journal of Fluid Mechanics, 631:281-309, 2009.

[11] L. D. Dailey, N. Meng, and R. H. Pletcher. Large eddy simulation of constant heat flux turbulent channel flow with property variations: quasi-developed model and mean flow results. Journal of Heat Transfer, 125(1):27-38, 2003.

[12] J. W. Deardorff. A numerical study of three-dimensional turbulent channel flow at large reynolds numbers. Journal of Fluid Mechanics, 41(2):453-480, 1970.

[13] F. Denaro, A. Abba, M. Germano, M. Icardi, D. Marchisio, S. Rolfo, P. Lampitella, E. Colombo, F. Inzoli, A. Aprovitola, F. S. Marra, M. Ioveno, and D. Tordella. A comparative test for assessing the performances of large-eddy simulation codes. In AIMETA Conference, 2011.

[14] J. A. Domaradzki, W. Liu, C. Hartel, and L. Kleiser. Energy transfer in numerically simulated wall-bounded turbulent flows. Physics of Fluids, 6(4):1583-1599, 1994.

[15] D. Dupuy, A. Toutant, and F. Bataille. Study of the sub-grid terms of the large-eddy simulation of a low Mach strongly anisothermal channel flow. In Eurotherm Seminar 106, Paris, France, 2016.

[16] D. Dupuy, A. Toutant, and F. Bataille. étude de l'équation d'énergie pour le développement de modèles sous-mailles adaptés aux écoulements fortement anisothermes. In Congrès SFT, Marseille, France, 2017.

[17] D. Dupuy, A. Toutant, and F. Bataille. Equations of energy exchanges in variable density turbulent flows. Physics Letters A, 382(5):327-333, 2018.

[18] D. Dupuy, A. Toutant, and F. Bataille. Study of the large-eddy simulation subgrid terms of a low mach number anisothermal channel flow. International Journal of Thermal Sciences, 135:221-234, 2018.

[19] D. Dupuy, A. Toutant, and F. Bataille. Turbulence kinetic energy exchanges in flows with highly variable fluid properties. Journal of Fluid Mechanics, 834:5-54, 2018.

[20] D. Dupuy, A. Toutant, and F. Bataille. A priori tests of subgrid-scale models in an anisothermal turbulent channel flow at low mach number. International Journal of Thermal Sciences, 145:105999, 2019.

[21] D. Dupuy, A. Toutant, and F. Bataille. Effect of the reynolds number on turbulence kinetic energy exchanges in flows with highly variable fluid properties. Physics of Fluids, 31(1): $015104,2019$. 
[22] D. Dupuy, A. Toutant, and F. Bataille. A posteriori tests of subgrid-scale models in an isothermal turbulent channel flow. Physics of Fluids, 31(4):045105, 2019.

[23] M. Germano, U. Piomelli, P. Moin, and W. H. Cabot. A dynamic subgrid-scale eddy viscosity model. Physics of Fluids A: Fluid Dynamics, 3(7):1760-1765, 1991.

[24] N. S. Ghaisas and S. H. Frankel. Dynamic gradient models for the sub-grid scale stress tensor and scalar flux vector in large eddy simulation. Journal of Turbulence, 17(1):30-50, 2016.

[25] T. Iliescu and P. Fischer. Large eddy simulation of turbulent channel flows by the rational large eddy simulation model. Physics of Fluids, 15(10):3036-3047, 2003.

[26] H. Jeanmart and G. Winckelmans. Investigation of eddy-viscosity models modified using discrete filters: a simplified "regularized variational multiscale model" and an "enhanced field model". Physics of Fluids, 19(5):055110, 2007.

[27] Z. Jiang, Z. Xia, Y. Shi, and S. Chen. Large-eddy simulation of plane channel flow with vreman's model. Journal of Turbulence, 17(8):807-822, 2016.

[28] J. Jiménez. The physics of wall turbulence. Physica A: Statistical Mechanics and its Applications, 263(1-4):252-262, 1999.

[29] J. Jiménez. Near-wall turbulence. Physics of Fluids, 25(10):101302, 2013.

[30] H. Kobayashi. The subgrid-scale models based on coherent structures for rotating homogeneous turbulence and turbulent channel flow. Physics of Fluids, 17(4):045104, 2005.

[31] P. Lampitella. Large eddy simulation for complex industrial flows. PhD thesis, Italy, 2014.

[32] J. Lee, H. Choi, and N. Park. Dynamic global model for large eddy simulation of transient flow. Physics of Fluids, 22(7):075106, 2010.

[33] A. Leonard. Energy cascade in large eddy simulations of turbulent fluid flows. Advances in Geophysics, 18A:237-248, 1974.

[34] B. Lessani and M. V. Papalexandris. Time-accurate calculation of variable density flows with strong temperature gradients and combustion. Journal of Computational Physics, 212(1): 218-246, 2006.

[35] B. Lessani and M. V. Papalexandris. Numerical study of turbulent channel flow with strong temperature gradients. International Journal of Numerical Methods for Heat $\&$ Fluid Flow, 18(3/4):545-556, 2008.

[36] D. K. Lilly. A proposed modification of the germano subgrid-scale closure method. Physics of Fluids A: Fluid Dynamics, 4(3):633-635, 1992.

[37] J. L. Lumley. Spectral energy budget in wall turbulence. Physics of Fluids, 7(2):190-196, 1964.

[38] J. Meyers and P. Sagaut. Is plane-channel flow a friendly case for the testing of large-eddy simulation subgrid-scale models? Physics of Fluids, 19(4):048105, 2007.

[39] Y. Morinishi and O. V. Vasilyev. A recommended modification to the dynamic two-parameter mixed subgrid scale model for large eddy simulation of wall bounded turbulent flow. Physics of Fluids, 13(11):3400-3410, 2001.

[40] Y. Morinishi, T. S. Lundhomas, O. V. Vasilyev, and P. Moin. Fully conservative higher order finite difference schemes for incompressible flow. Journal of Computational Physics, 143(1): 90-124, 1998.

[41] F. Nicoud. Conservative high-order finite-difference schemes for low-Mach number flows. Journal of Computational Physics, 158(1):71-97, 2000.

[42] F. Nicoud and F. Ducros. Subgrid-scale stress modelling based on the square of the velocity gradient tensor. Flow, Turbulence and Combustion, 62(3):183-200, 1999. ISSN 1386-6184.

[43] F. Nicoud, H. Baya Toda, O. Cabrit, S. Bose, and J. Lee. Using singular values to build a subgrid-scale model for large eddy simulations. Physics of Fluids, 23(8):085106, 2011.

[44] F. C. Nicoud. Numerical study of a channel flow with variable properties. Center for Turbulence Research Annual Research Briefs, pages 289-309, 1998.

[45] T. Ohtsuka and K. Abe. Toward the development of an anisotropy-resolving subgrid-scale model for large eddy simulation. Journal of Fluid Science and Technology, 9(1):JFST0004JFST0004, 2014.

[46] N. Park, S. Lee, J. Lee, and H. Choi. A dynamic subgrid-scale eddy viscosity model with a global model coefficient. Physics of Fluids, 18(12):125109, 2006.

[47] A. Patel, J. Peeters, B. Boersma, and R. Pecnik. Semi-local scaling and turbulence modulation in variable property turbulent channel flows. Physics of Fluids, 27(9):095101, 2015. 
[48] A. Patel, B. J. Boersma, and R. Pecnik. Scalar statistics in variable property turbulent channel flows. Physical Review Fluids, 2(8):084604, 2017.

[49] M. Rieth, F. Proch, O. T. Stein, M. W. A. Pettit, and A. M. Kempf. Comparison of the sigma and smagorinsky les models for grid generated turbulence and a channel flow. Computers 83 Fluids, 99:172-181, 2014.

[50] W. Rozema, H.J. Bae, P. Moin, and R. Verstappen. Minimum-dissipation models for largeeddy simulation. Physics of Fluids, 27(8):085107, 2015.

[51] S. Ryu and G. Iaccarino. A subgrid-scale eddy-viscosity model based on the volumetric strainstretching. Physics of Fluids, 26(6):065107, 2014.

[52] P. Sagaut. Large eddy simulation for incompressible flows: an introduction. Springer Science \& Business Media, 2006.

[53] M. Sanchez, F. Aulery, A. Toutant, and F. Bataille. Large eddy simulation of thermal boundary layer spatial development in a turbulent channel flow. Journal of Fluids Engineering, 136 (6):060906, 2014.

[54] S. Serra, A. Toutant, and F. Bataille. Thermal large eddy simulation in a very simplified geometry of a solar receiver. Heat Transfer Engineering, 33(6):505-524, 2012.

[55] S. Serra, A. Toutant, F. Bataille, and Y. Zhou. High-temperature gradient effect on a turbulent channel flow using thermal large-eddy simulation in physical and spectral spaces. Journal of Turbulence, 13:N49, 2012.

[56] S. Serra, A. Toutant, F. Bataille, and Y. Zhou. Turbulent kinetic energy spectrum in very anisothermal flows. Physics Letters A, 376(45):3177-3184, 2012.

[57] M. H. Silvis, R. A. Remmerswaal, and R. Verstappen. Physical consistency of subgrid-scale models for large-eddy simulation of incompressible turbulent flows. Physics of Fluids, 29(1): 015105, 2017.

[58] S. Singh and D. You. A dynamic global-coefficient mixed subgrid-scale model for large-eddy simulation of turbulent flows. International Journal of Heat and Fluid Flow, 42:94-104, 2013.

[59] J. Smagorinsky. General circulation experiments with the primitive equations: I. the basic experiment. Monthly Weather Review, 91(3):99-164, 1963.

[60] S. Stolz, N. A. Adams, and L. Kleiser. An approximate deconvolution model for large-eddy simulation with application to incompressible wall-bounded flows. Physics of Fluids, 13(4): 997-1015, 2001.

[61] W. Sutherland. The viscosity of gases and molecular force. The London, Edinburgh, and Dublin Philosophical Magazine and Journal of Science, 36(223):507-531, 1893.

[62] A. Toutant and F. Bataille. Turbulence statistics in a fully developed channel flow submitted to a high temperature gradient. International Journal of Thermal Sciences, 74:104-118, 2013.

[63] A. Trettel and J. Larsson. Mean velocity scaling for compressible wall turbulence with heat transfer. Physics of Fluids, 28(2):026102, 2016.

[64] F. X. Trias, D. Folch, A. Gorobets, and A. Oliva. Building proper invariants for eddy-viscosity subgrid-scale models. Physics of Fluids, 27(6):065103, 2015.

[65] R. Verstappen. When does eddy viscosity damp subfilter scales sufficiently? Journal of Scientific Computing, 49(1):94, 2011.

[66] A. W. Vreman. An eddy-viscosity subgrid-scale model for turbulent shear flow: Algebraic theory and applications. Physics of Fluids, 16(10):3670-3681, 2004.

[67] J. H. Williamson. Low-storage Runge-Kutta schemes. Journal of Computational Physics, 35 (1):48-56, 1980.

[68] D. You and P. Moin. A dynamic global-coefficient subgrid-scale eddy-viscosity model for large-eddy simulation in complex geometries. Physics of Fluids, 19(6):065110, 2007.

[69] C. Yu, Z. Xiao, and X. Li. Scale-adaptive subgrid-scale modelling for large-eddy simulation of turbulent flows. Physics of Fluids, 29(3):035101, 2017.

[70] Y. Zhou. Rayleigh-taylor and richtmyer-meshkov instability induced flow, turbulence, and mixing. i. Physics Reports, 720-722:1-136, 2017.

[71] Y. Zhou. Rayleigh-taylor and richtmyer-meshkov instability induced flow, turbulence, and mixing. ii. Physics Reports, 723-725:1-160, 2017. 

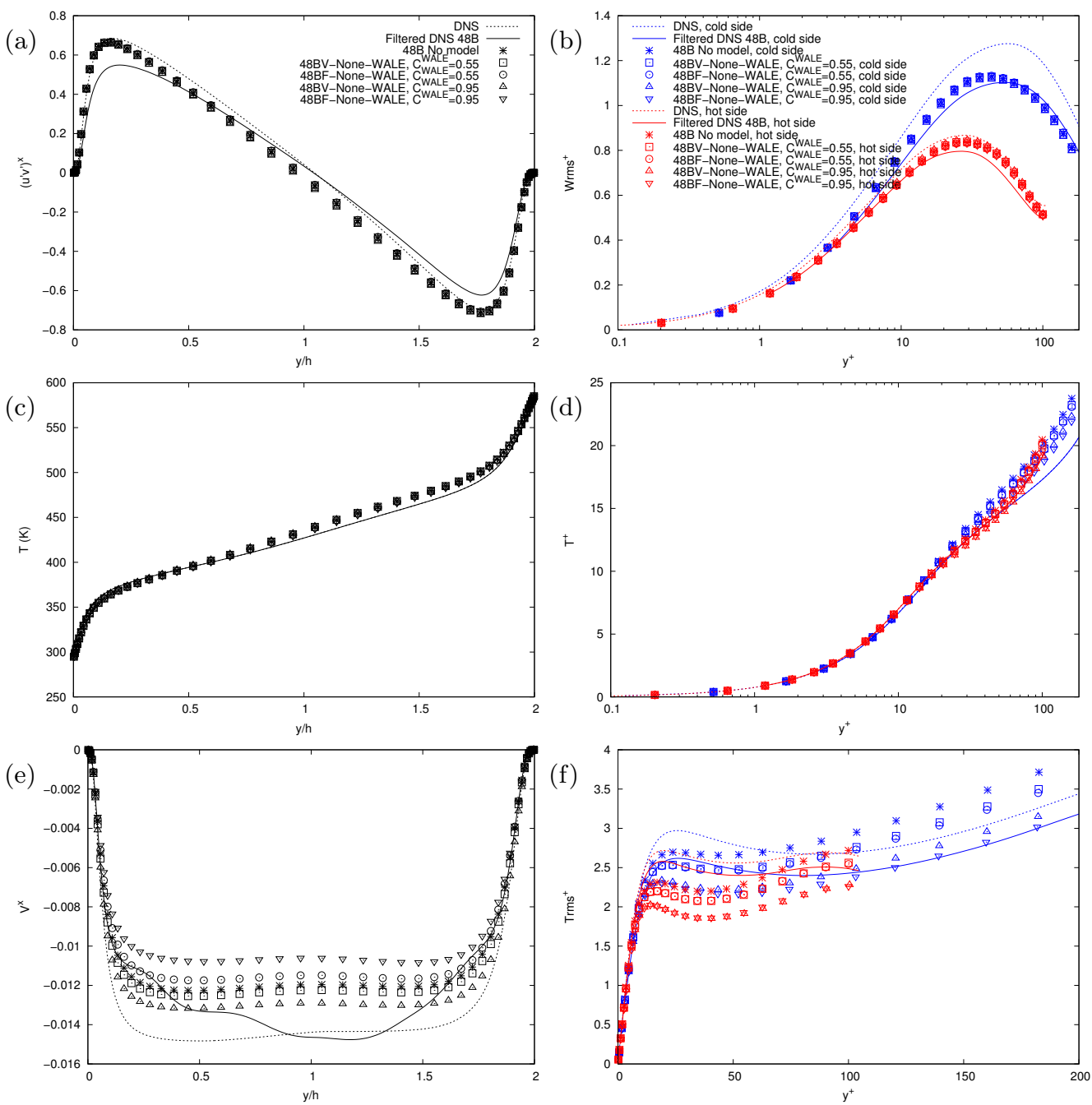

Figure 11 - (Colour online) Comparison of large-eddy simulations with the WALE model for the density-velocity correlation subgrid term in the Velocity and Favre formulations for the profiles of the covariance of streamwise and wall-normal velocity $\left\langle u_{x}^{\prime} u_{y}^{\prime}\right\rangle$ (a), the standard deviation of spanwise velocity $\sqrt{\left\langle u_{z}^{\prime 2}\right\rangle}(\mathrm{b})$, the mean temperature $\langle T\rangle(\mathrm{c}, \mathrm{d})$, the mean wall-normal velocity $\left\langle U_{y}\right\rangle$ (e) and the standard deviation of temperature $\sqrt{\left\langle T^{\prime 2}\right\rangle}$ (f) at $R e_{\tau}=180$ with the mesh $48 \mathrm{~B}$. 

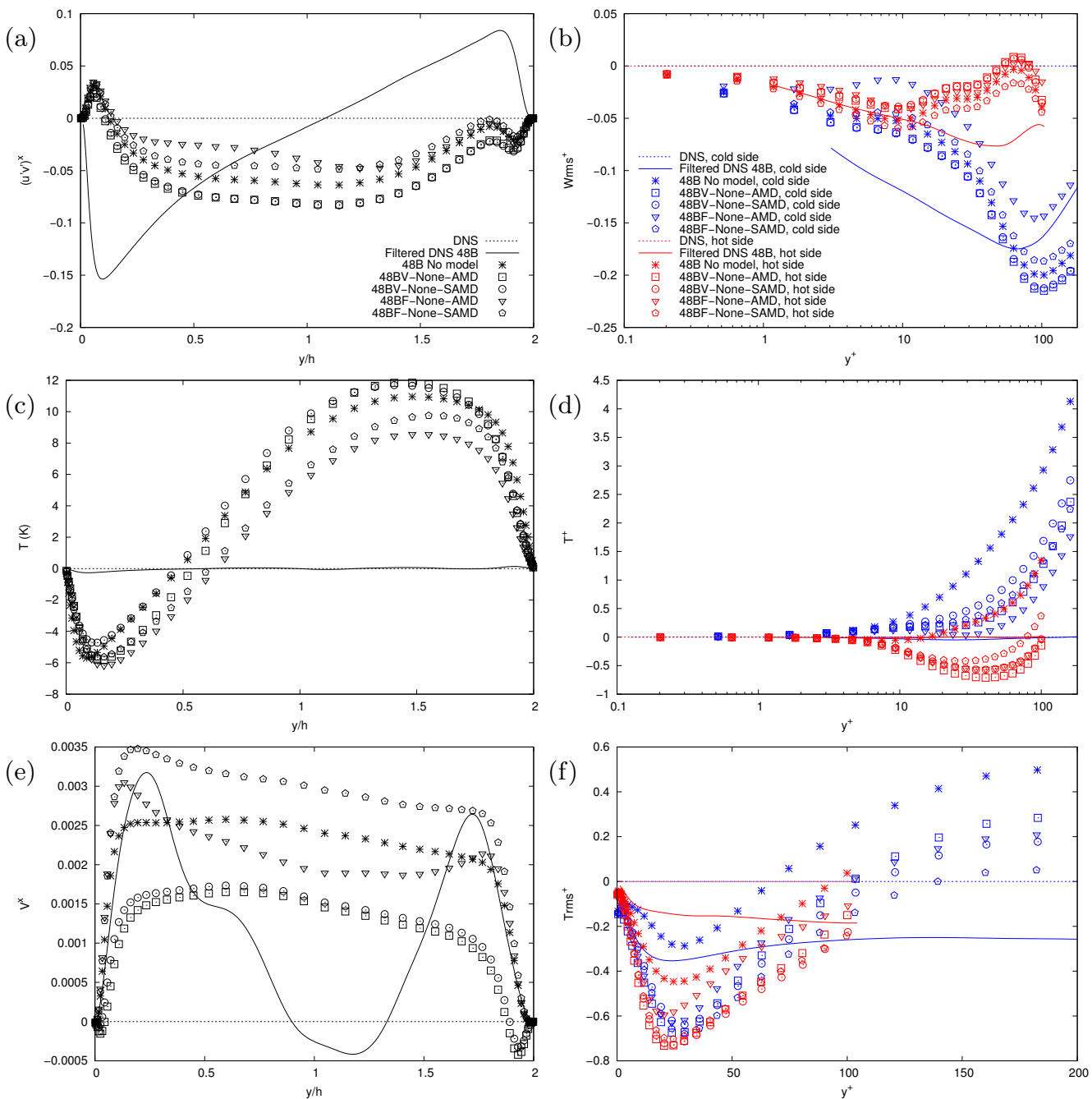

Figure 12 - (Colour online) Comparison of large-eddy simulations with the AMD and scalar AMD (SAMD) model for the density-velocity correlation subgrid term in the Velocity and Favre formulations for the profiles of the covariance of streamwise and wall-normal velocity $\left\langle u_{x}^{\prime} u_{y}^{\prime}\right\rangle$ (a), the standard deviation of spanwise velocity $\sqrt{\left\langle u_{z}^{\prime 2}\right\rangle}$ (b), the mean temperature $\langle T\rangle$ (c, d), the mean wall-normal velocity $\left\langle U_{y}\right\rangle$ (e) and the standard deviation of temperature $\sqrt{\left\langle T^{\prime 2}\right\rangle}$ (f) at $R e_{\tau}=180$ with the mesh 48B. For clarity, the profiles are presented as differences from the baseline nonfiltered direct numerical simulation. 

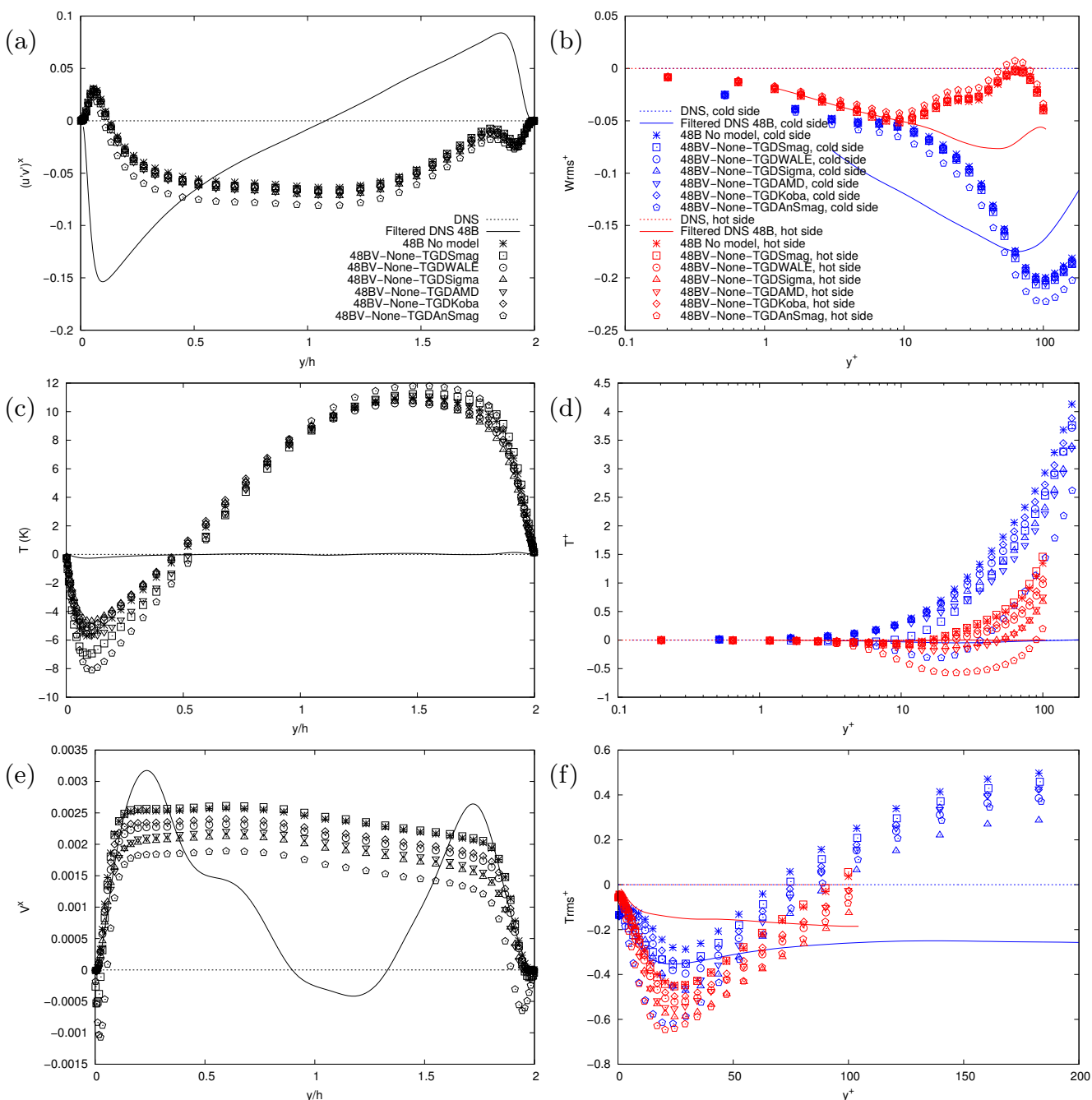

Figure 13 - (Colour online) Comparison of large-eddy simulations with the vectorial globalaverage dynamic Smagorinsky (TGDSmag), WALE (TGDWALE), Sigma (TGDSigma), AMD (TGDAMD), Kobayashi (TGDKoba) and Anisotropic Smagorinsky (TGDAnSmag) models for the density-velocity correlation subgrid term in the Velocity formulation for the profiles of the covariance of streamwise and wall-normal velocity $\left\langle u_{x}^{\prime} u_{y}^{\prime}\right\rangle$ (a), the standard deviation of spanwise velocity $\sqrt{\left\langle u_{z}^{\prime 2}\right\rangle}$ (b), the mean temperature $\langle T\rangle$ (c, d), the mean wall-normal velocity $\left\langle U_{y}\right\rangle$ (e) and the standard deviation of temperature $\sqrt{\left\langle T^{\prime 2}\right\rangle}$ (f) at $R e_{\tau}=180$ with the mesh 48B. For clarity, the profiles are presented as differences from the baseline nonfiltered direct numerical simulation. 

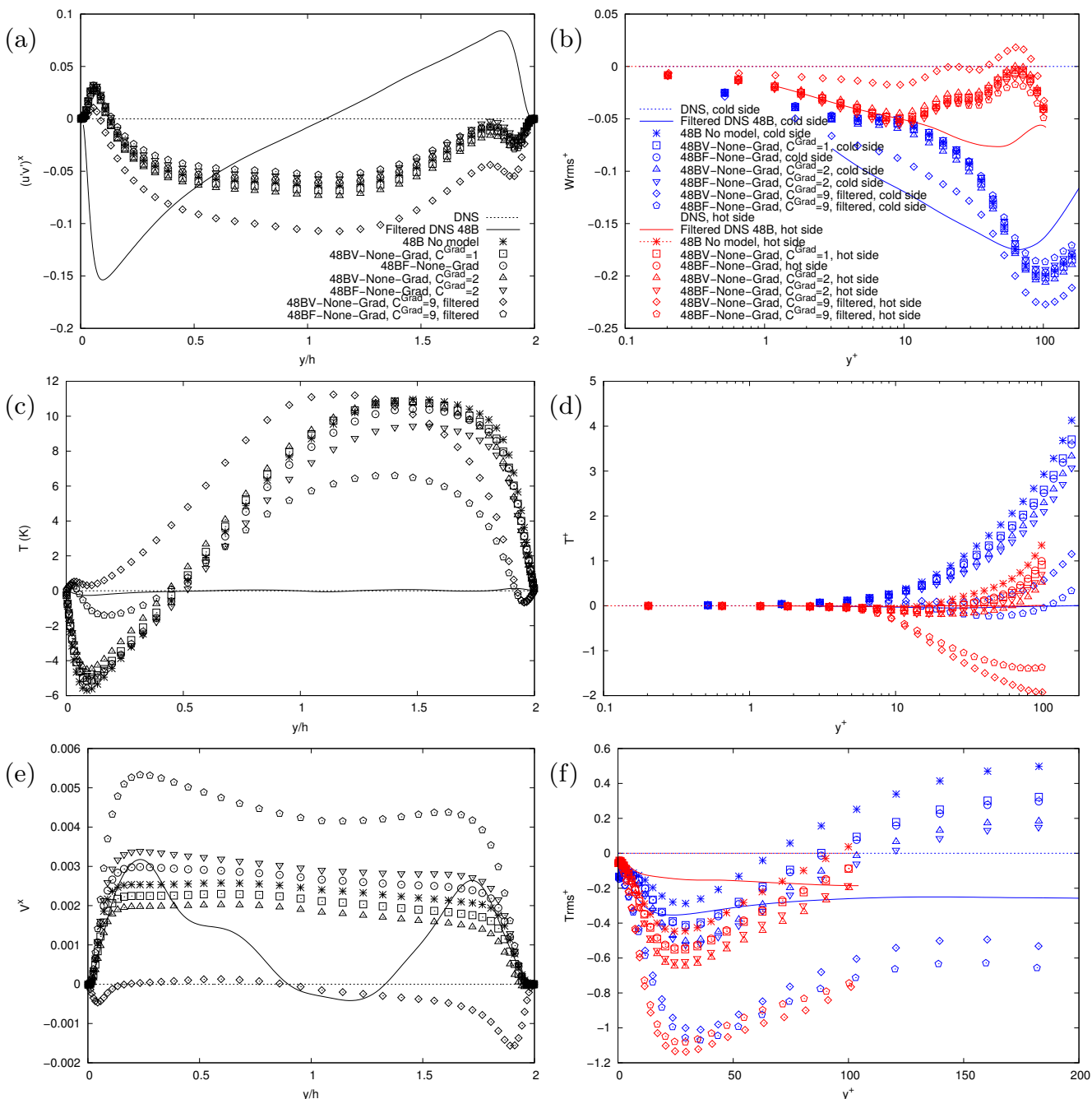

Figure 14 - (Colour online) Comparison of large-eddy simulations with the gradient model using $C^{\text {Grad. }}=1$ and $C^{\text {Grad. }}=2$ and the filtered gradient model using $C^{\text {Grad. }}=2$ and $C^{\text {Grad. }}=9$ for the density-velocity correlation subgrid term in the Velocity formulation for the profiles of the covariance of streamwise and wall-normal velocity $\left\langle u_{x}^{\prime} u_{y}^{\prime}\right\rangle$ (a), the standard deviation of spanwise velocity $\sqrt{\left\langle u_{z}^{\prime 2}\right\rangle}(\mathrm{b})$, the mean temperature $\langle T\rangle(\mathrm{c}, \mathrm{d})$, the mean wall-normal velocity $\left\langle U_{y}\right\rangle$ (e) and the standard deviation of temperature $\sqrt{\left\langle T^{\prime 2}\right\rangle}$ (f) at $R e_{\tau}=180$ with the mesh $48 \mathrm{~B}$. For clarity, the profiles are presented as differences from the baseline nonfiltered direct numerical simulation. 

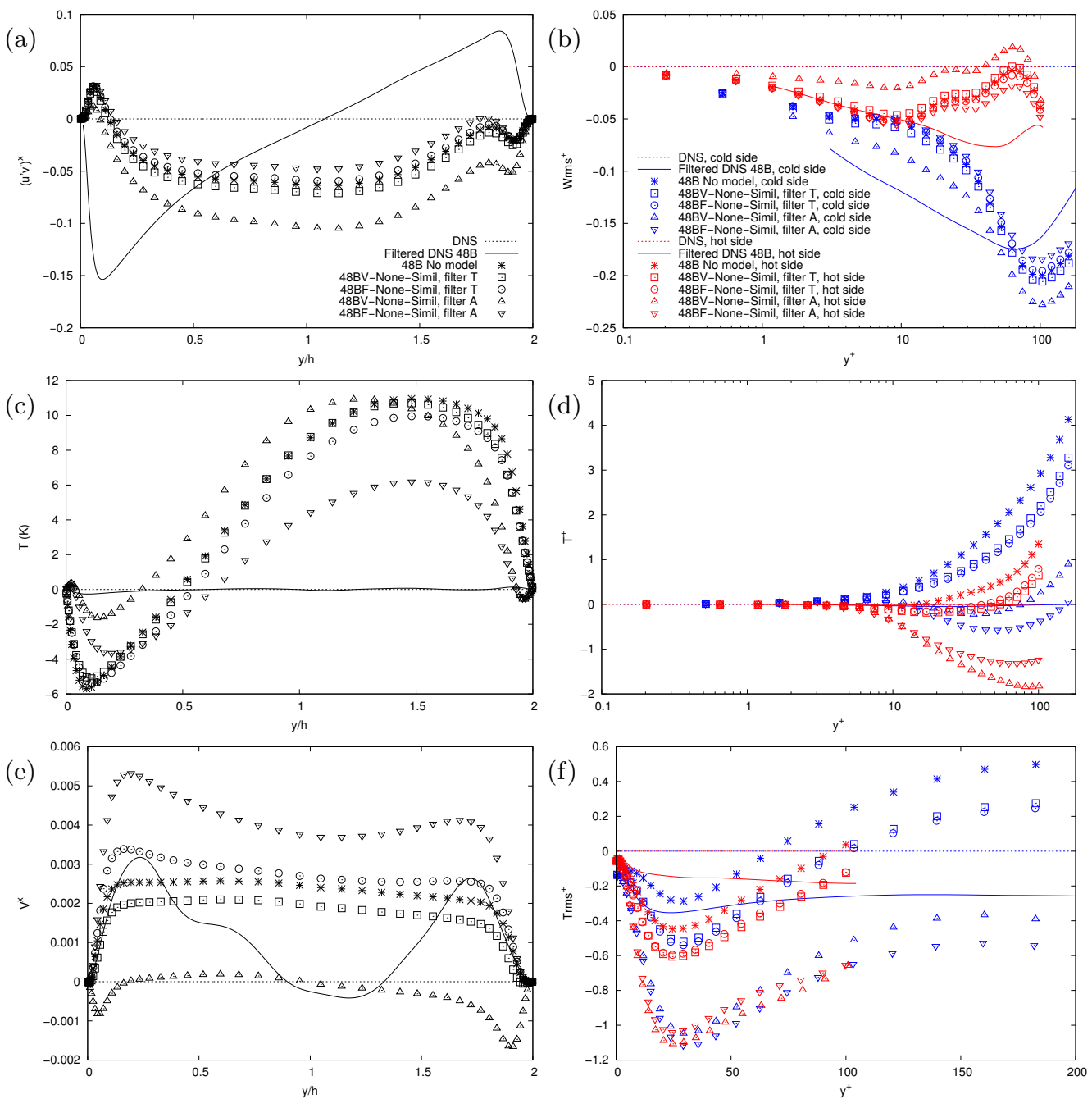

Figure 15 - (Colour online) Comparison of large-eddy simulations with the scale-similarity model using filter $\mathrm{T}$ and filter A for the density-velocity correlation subgrid term in the Velocity formulation for the profiles of the covariance of streamwise and wall-normal velocity $\left\langle u_{x}^{\prime} u_{y}^{\prime}\right\rangle$ (a), the standard deviation of spanwise velocity $\sqrt{\left\langle u_{z}^{\prime 2}\right\rangle}$ (b), the mean temperature $\langle T\rangle(\mathrm{c}, \mathrm{d})$, the mean wall-normal velocity $\left\langle U_{y}\right\rangle$ (e) and the standard deviation of temperature $\sqrt{\left\langle T^{\prime 2}\right\rangle}$ (f) at $R e_{\tau}=180$ with the mesh 48B. For clarity, the profiles are presented as differences from the baseline nonfiltered direct numerical simulation. 


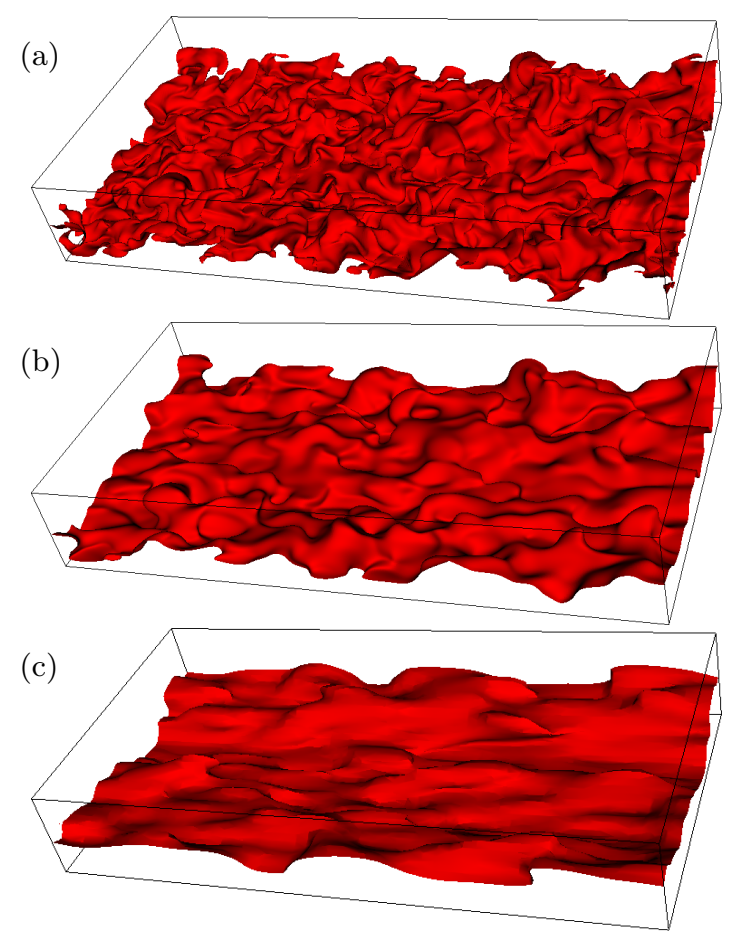

Figure 16 - Isosurface of the temperature $400 \mathrm{~K}$ for the nonfiltered direct numerical simulation (a), the filtered direct numerical simulation (b) and the large-eddy simulation with the $H^{(4)}$ AMD model for the momentum convection subgrid term and the scale-similarity model for the density-velocity correlation subgrid term in the Velocity formulation at $R e_{\tau}=180$ (c). Note that in this figure the filtered direct numerical simulation is sampled at the same resolution as the nonfiltered direct numerical simulation. 
(a)

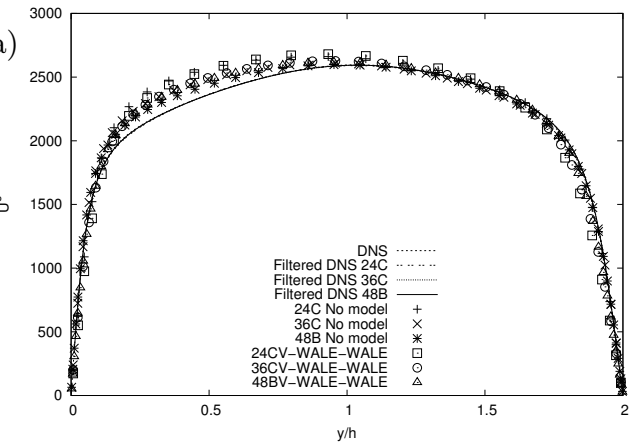

(c)
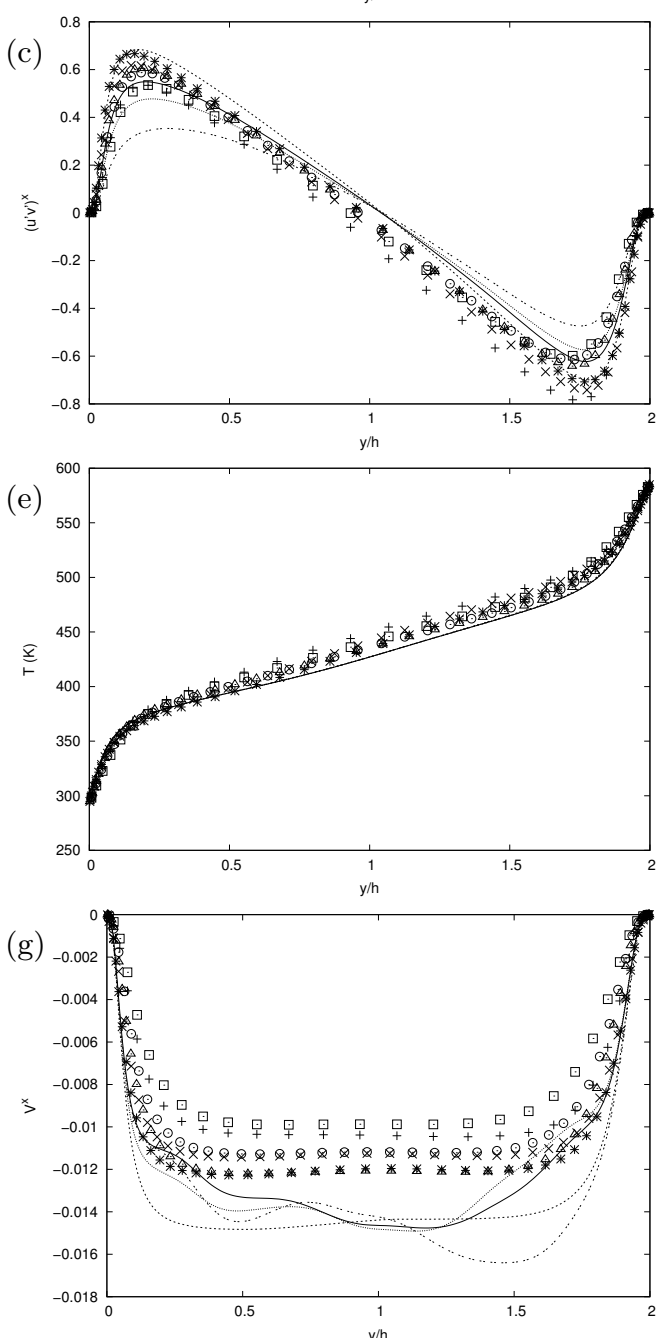
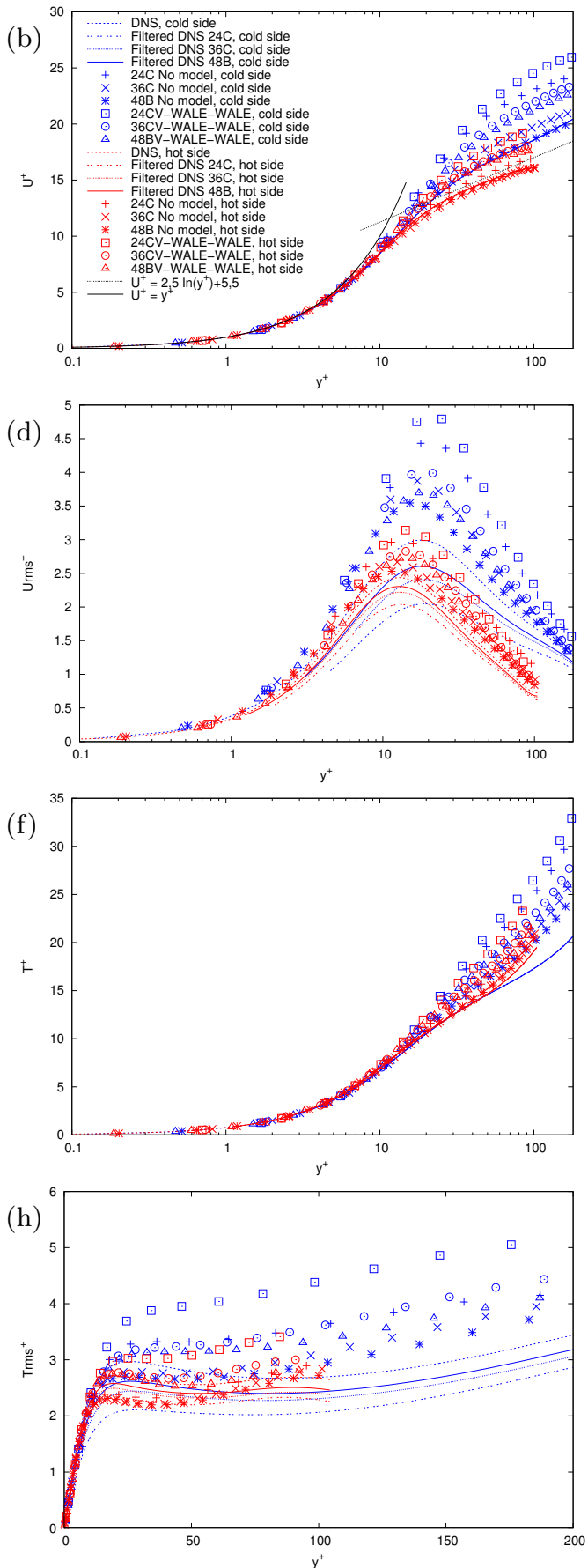

Figure 17 - (Colour online) Comparison of large-eddy simulations with the WALE model for the momentum convection subgrid term and the density-velocity correlation subgrid term with the meshes $24 \mathrm{C}, 36 \mathrm{C}$ and $48 \mathrm{~B}$ in the Velocity formulation for the profiles of the mean streamwise velocity $\left\langle U_{x}\right\rangle(\mathrm{a}, \mathrm{b})$, the covariance of streamwise and wall-normal velocity $\left\langle u_{x}^{\prime} u_{y}^{\prime}\right\rangle$ (c), the standard deviation of streamwise velocity $\sqrt{\left\langle u_{x}^{\prime 2}\right\rangle}$ (d), the mean temperature $\langle T\rangle$ (e, f), the mean wall-normal velocity $\left\langle U_{y}\right\rangle$ (g) and the standard deviation of temperature $\sqrt{\left\langle T^{\prime 2}\right\rangle}(\mathrm{h})$ at $R e_{\tau}=180$. 
(a)

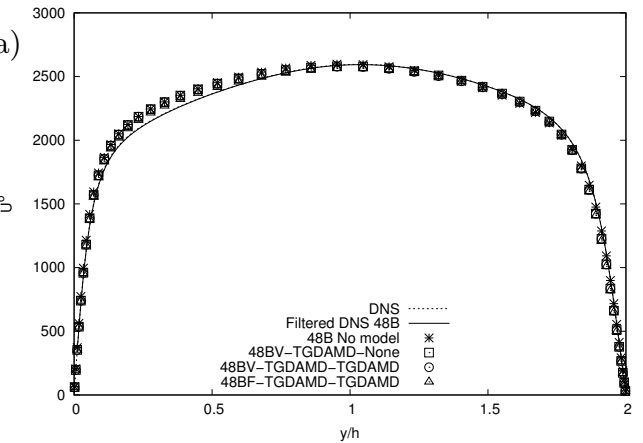

(c)

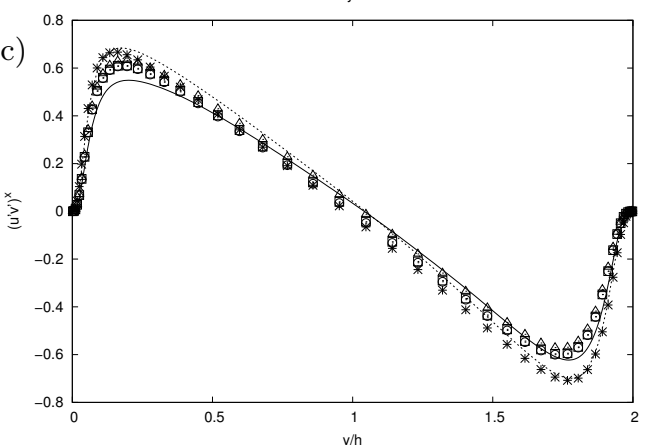

(e)
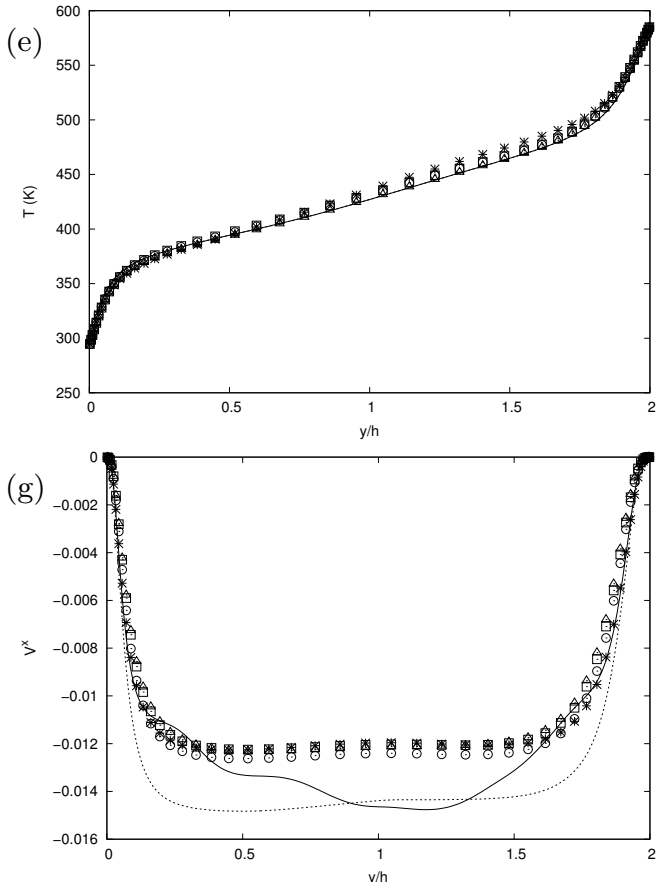

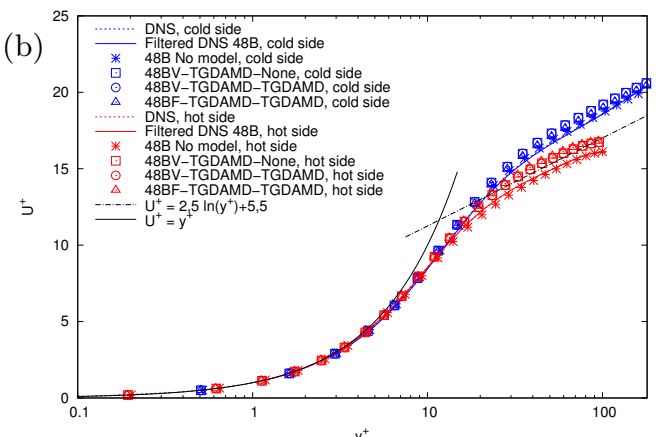

(d)
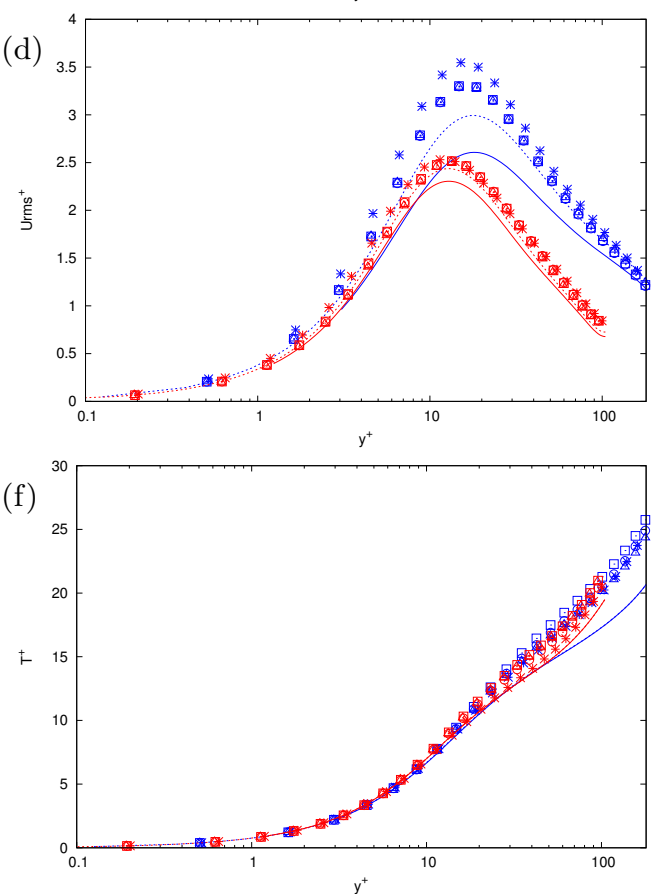

(h)

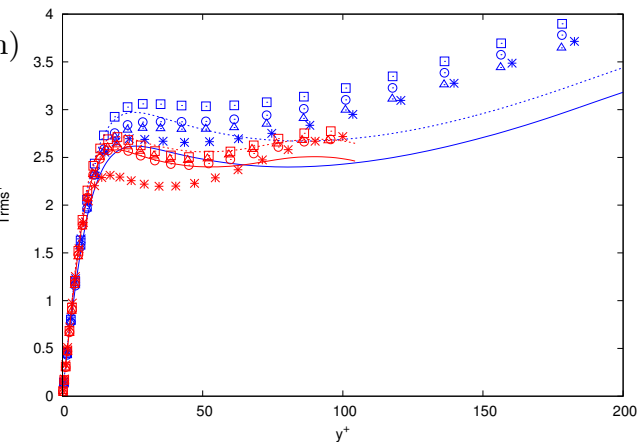

Figure 18 - (Colour online) Comparison of large-eddy simulations with the tensorial/vectorial global-average dynamic AMD (TGDAMD) model for either the momentum convection subgrid term alone or both the momentum convection subgrid term and the densityvelocity correlation subgrid term in the Velocity and Favre formulations for the profiles of the mean streamwise velocity $\left\langle U_{x}\right\rangle(\mathrm{a}, \mathrm{b})$, the covariance of streamwise and wall-normal velocity $\left\langle u_{x}^{\prime} u_{y}^{\prime}\right\rangle$ (c), the standard deviation of streamwise velocity $\sqrt{\left\langle u_{x}^{\prime 2}\right\rangle}$ (d), the mean temperature $\langle T\rangle$ (e,f), the mean wall-normal velocity $\left\langle U_{y}\right\rangle$ (g) and the standard deviation of temperature $\sqrt{\left\langle T^{\prime 2}\right\rangle}(\mathrm{h})$ at $R e_{\tau}=180$ with the mesh $48 \mathrm{~B}$. 

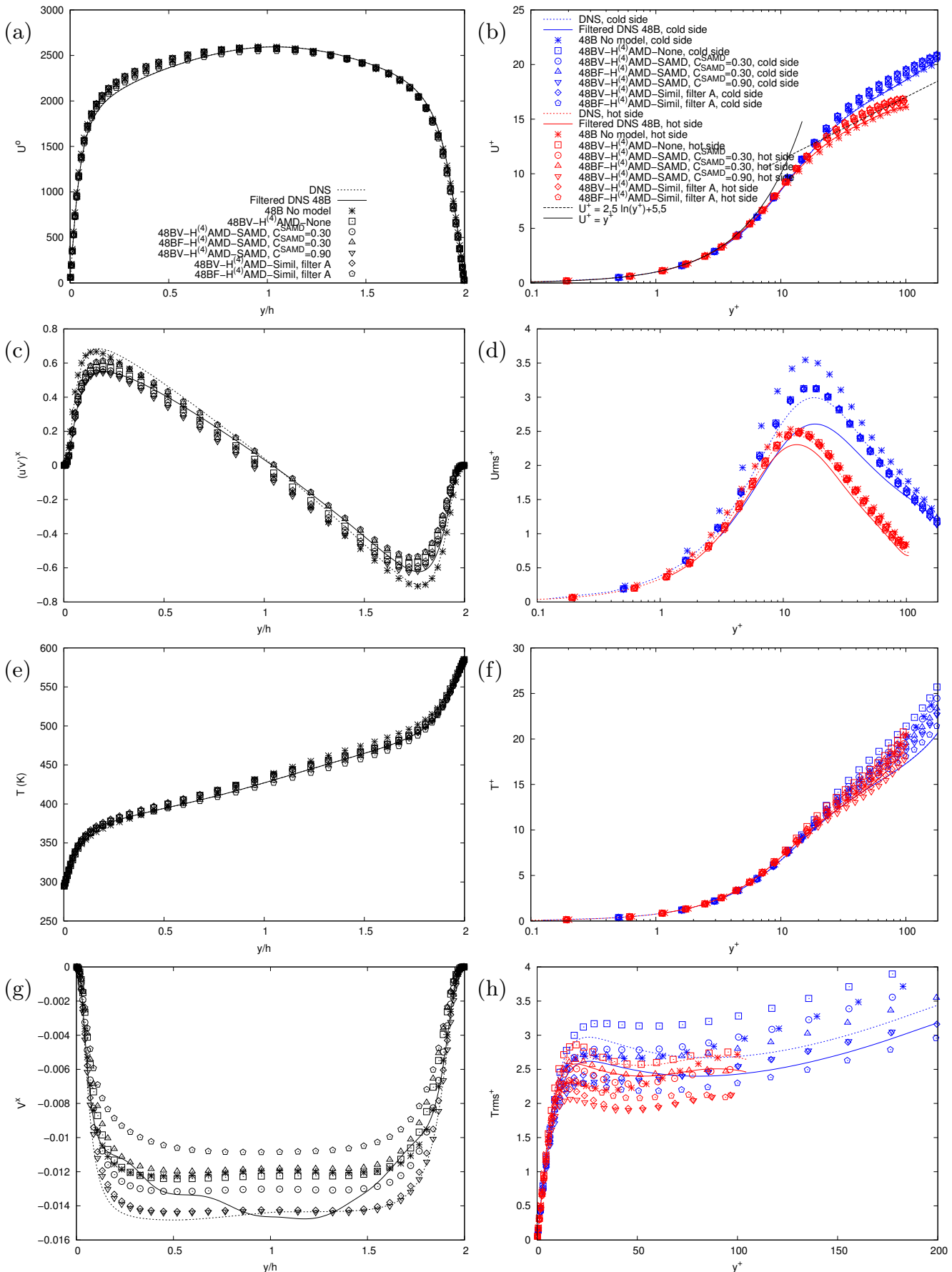

Figure 19 - (Colour online) Comparison of large-eddy simulations with the tensorial AMD model based on the $H^{(4)}$ tensor $\left(H^{(4)} \mathrm{AMD}\right)$ for the momentum convection subgrid term and the scalar AMD model (SAMD) or the scale-similarity model (Simil) using filter A for the density-velocity correlation subgrid term in the Velocity and Favre formulations for the profiles of the mean streamwise velocity $\left\langle U_{x}\right\rangle(\mathrm{a}, \mathrm{b})$, the covariance of streamwise and wall-normal velocity $\left\langle u_{x}^{\prime} u_{y}^{\prime}\right\rangle$ (c), the standard deviation of streamwise velocity $\sqrt{\left\langle u_{x}^{\prime 2}\right\rangle}$ (d), the mean temperature $\langle T\rangle$ (e, f), the mean wall-normal velocity $\left\langle U_{y}\right\rangle$ (g) and the standard deviation of temperature $\sqrt{\left\langle T^{\prime 2}\right\rangle}(\mathrm{h})$ at $R e_{\tau}=180$ with the mesh 48B. 
(a)

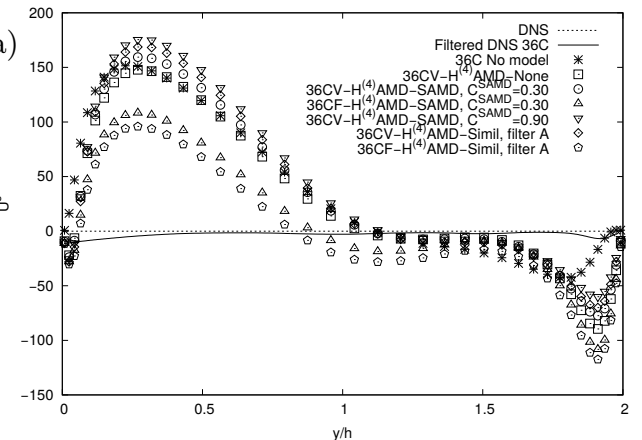

(c)

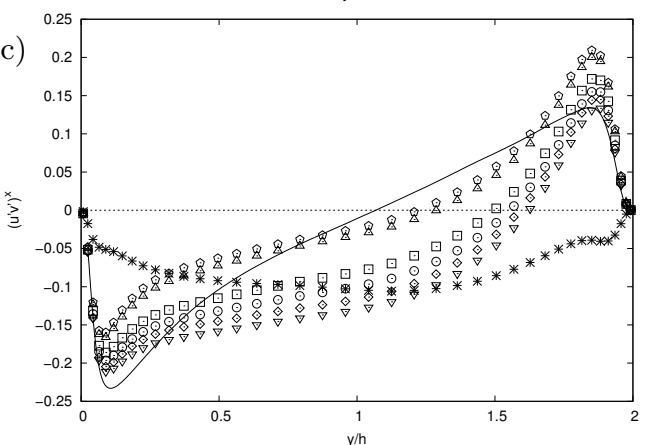

(e)
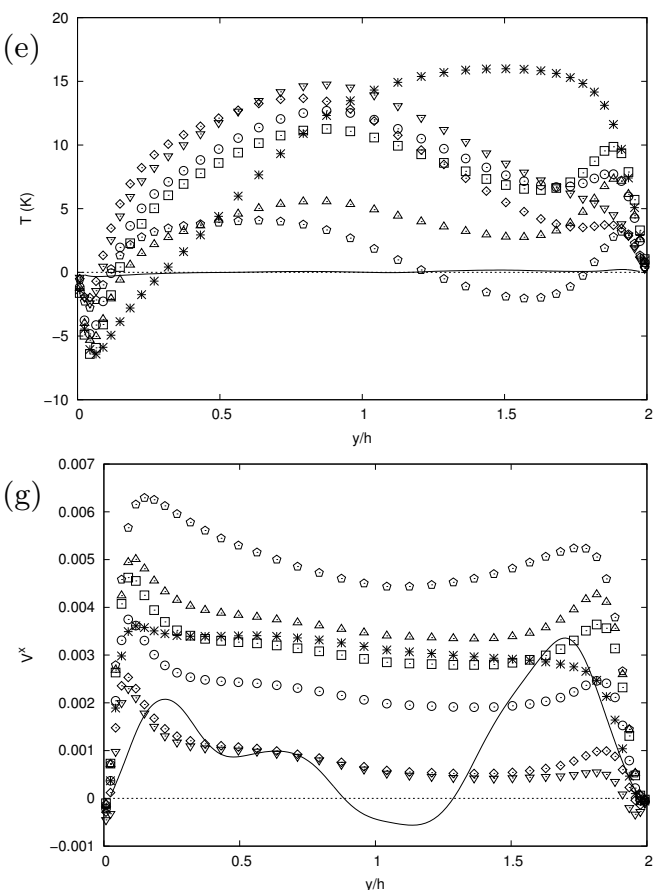
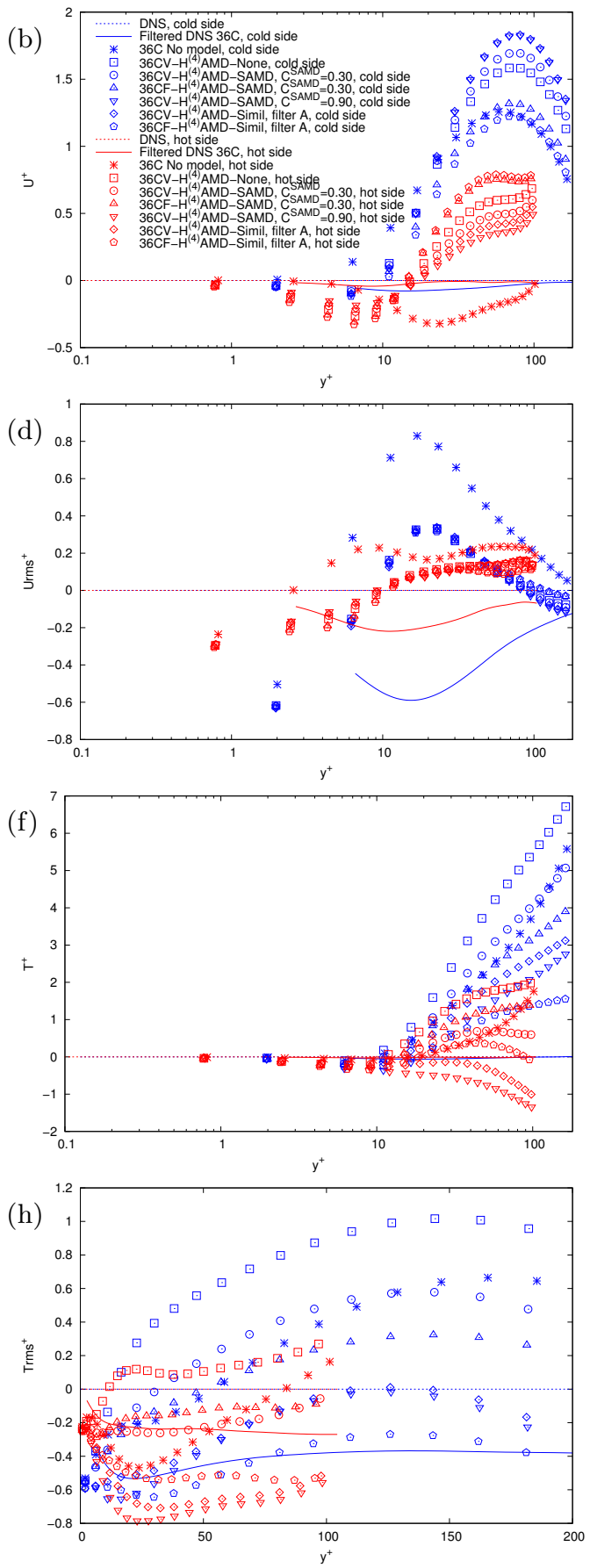

Figure 20 - (Colour online) Comparison of large-eddy simulations with the tensorial AMD model based on the $H^{(4)}$ tensor $\left(H^{(4)} \mathrm{AMD}\right)$ for the momentum convection subgrid term and the scalar AMD model (SAMD) or the scale-similarity model (Simil) using filter A for the density-velocity correlation subgrid term in the Velocity and Favre formulations for the profiles of the mean streamwise velocity $\left\langle U_{x}\right\rangle(\mathrm{a}, \mathrm{b})$, the covariance of streamwise and wall-normal velocity $\left\langle u_{x}^{\prime} u_{y}^{\prime}\right\rangle$ (c), the standard deviation of streamwise velocity $\sqrt{\left\langle u_{x}^{\prime 2}\right\rangle}$ (d), the mean temperature $\langle T\rangle$ (e, f), the mean wall-normal velocity $\left\langle U_{y}\right\rangle$ (g) and the standard deviation of temperature $\sqrt{\left\langle T^{\prime 2}\right\rangle}(\mathrm{h})$ at $R e_{\tau}=180$ with the mesh 36C. For clarity, the profiles are presented as differences from the baseline nonfiltered direct numerical simulation. 

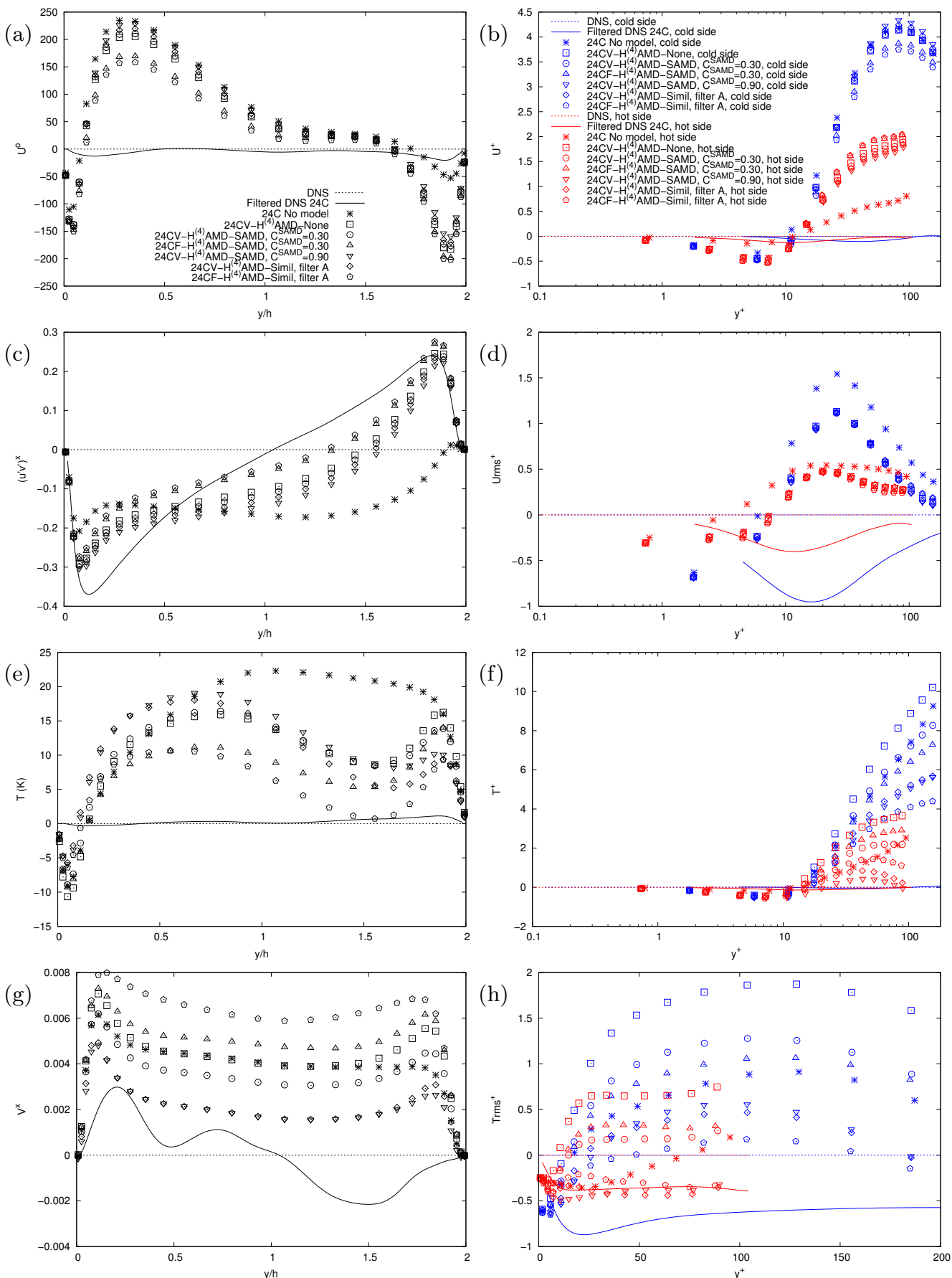

Figure 21 - (Colour online) Comparison of large-eddy simulations with the tensorial AMD model based on the $H^{(4)}$ tensor $\left(H^{(4)} \mathrm{AMD}\right)$ for the momentum convection subgrid term and the scalar AMD model (SAMD) or the scale-similarity model (Simil) using filter A for the density-velocity correlation subgrid term in the Velocity and Favre formulations for the profiles of the mean streamwise velocity $\left\langle U_{x}\right\rangle(\mathrm{a}, \mathrm{b})$, the covariance of streamwise and wall-normal velocity $\left\langle u_{x}^{\prime} u_{y}^{\prime}\right\rangle$ (c), the standard deviation of streamwise velocity $\sqrt{\left\langle u_{x}^{\prime 2}\right\rangle}$ (d), the mean temperature $\langle T\rangle$ (e, f), the mean wall-normal velocity $\left\langle U_{y}\right\rangle$ (g) and the standard deviation of temperature $\sqrt{\left\langle T^{\prime 2}\right\rangle}$ (h) at $R e_{\tau}=180$ with the mesh 24C. For clarity, the profiles are presented as differences from the baseline nonfiltered direct numerical simulation. 

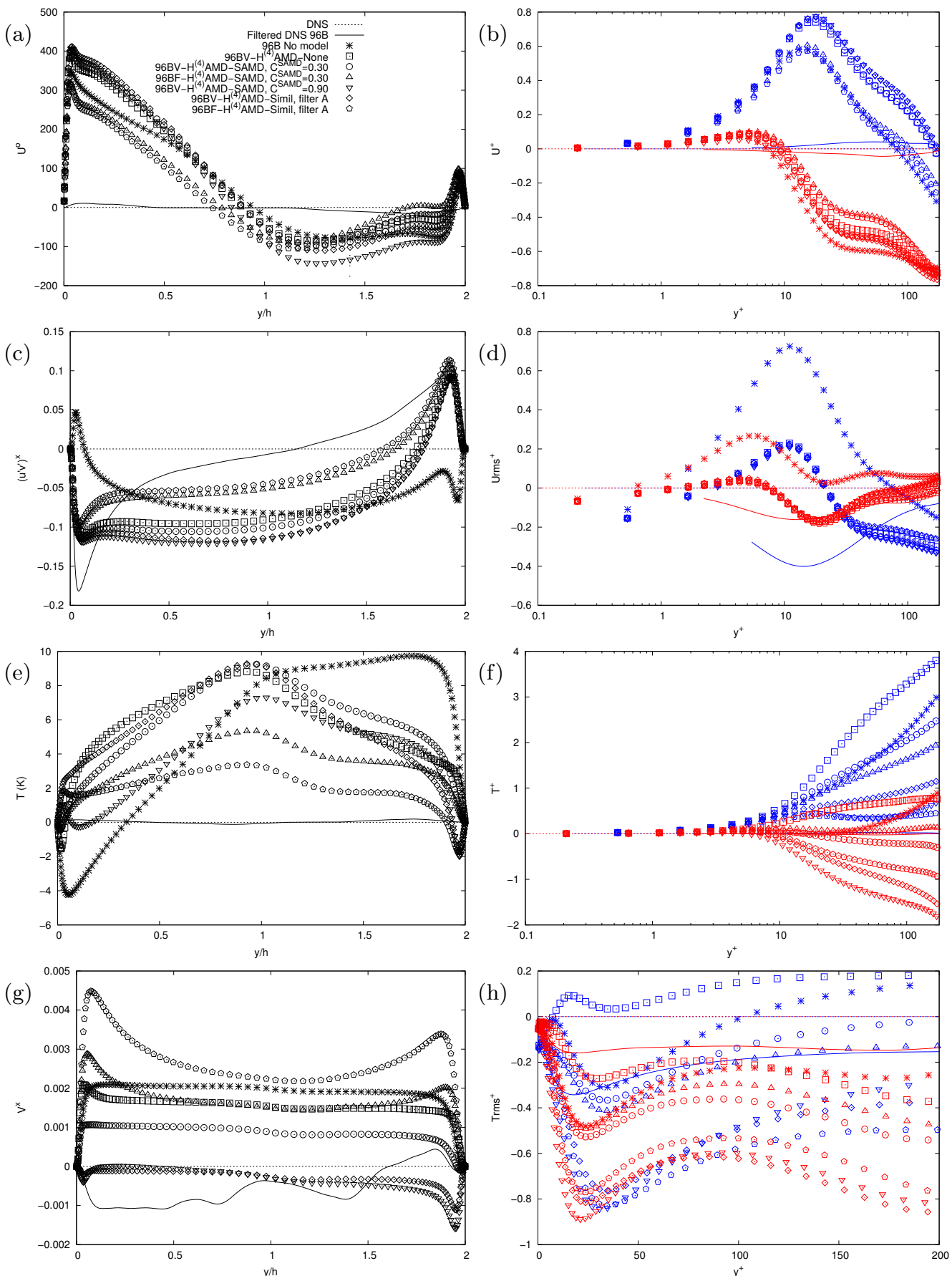

Figure 22 - (Colour online) Comparison of large-eddy simulations with the tensorial AMD model based on the $H^{(4)}$ tensor $\left(H^{(4)} \mathrm{AMD}\right)$ for the momentum convection subgrid term and the scalar AMD model (SAMD) or the scale-similarity model (Simil) using filter A for the density-velocity correlation subgrid term in the Velocity and Favre formulations for the profiles of the mean streamwise velocity $\left\langle U_{x}\right\rangle(\mathrm{a}, \mathrm{b})$, the covariance of streamwise and wall-normal velocity $\left\langle u_{x}^{\prime} u_{y}^{\prime}\right\rangle$ (c), the standard deviation of streamwise velocity $\sqrt{\left\langle u_{x}^{\prime 2}\right\rangle}$ (d), the mean temperature $\langle T\rangle$ (e, f), the mean wall-normal velocity $\left\langle U_{y}\right\rangle$ (g) and the standard deviation of temperature $\sqrt{\left\langle T^{\prime 2}\right\rangle}(\mathrm{h})$ at $R e_{\tau}=395$ with the mesh 96B. For clarity, the profiles are presented as differences from the baseline nonfiltered direct numerical simulation. 